Pontifícia Universidade Católica $_{\text {a }}$

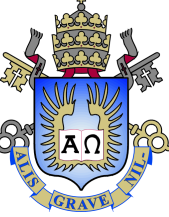

Daniel Lívio Alencar Cordeiro

\title{
Uma proxy para aversão ao risco avaliada no
} mercado de ações

Dissertação de Mestrado

Dissertação apresentada como requisito parcial para obtenção do grau de Mestre pelo Programa de Pós-graduação em Economia do Departamento de Economia do Centro de Ciências Sociais da PUC-Rio.

Orientador : Prof. Ruy Monteiro Ribeiro Coorientador: Prof. Eduardo Zilberman 


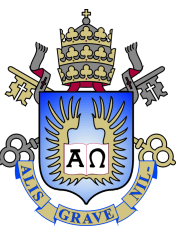

Daniel Lívio Alencar Cordeiro

\section{Uma proxy para aversão ao risco avaliada no mercado de ações}

Dissertação apresentada como requisito parcial para obtenção do grau de Mestre pelo Programa de Pós-graduação em Economia do Departamento de Economia do Centro de Ciências Sociais da PUC-Rio. Aprovada pela Comissão Examinadora abaixo assinada.

Prof. Ruy Monteiro Ribeiro Orientador Departamento de Economia - PUC-Rio

Prof. Eduardo Zilberman Coorientador

Pontifícia Universidade Católica do Rio de Janeiro - PUC-Rio

Prof. Marcelo Cunha Medeiros Departamento de Economia - PUC-Rio

Prof. João Victor Issler Escola Brasileira de Economia e Finanças - EPGE

Prof. Augusto Cesar Pinheiro da Silva Vice-Decano de Pós-Graduação do Centro de Ciências Sociais - PUC-Rio 
Todos os direitos reservados. É proibida a reprodução total ou parcial do trabalho sem autorização da universidade, do autor e do orientador.

\section{Daniel Lívio Alencar Cordeiro}

Graduou-se em Engenharia de Fortificação e Construção pelo Instituto Militar de Engenharia em 2012

Ficha Catalográfica

Cordeiro Alencar, Daniel Lívio

Uma proxy para aversão ao risco avaliada no mercado de ações / Daniel Lívio Alencar Cordeiro; orientador: Ruy Monteiro Ribeiro; co-orientador: Eduardo Zilberman. - 2017.

v., 41 f: il. color. ; $30 \mathrm{~cm}$

Dissertação (mestrado) - Pontifícia Universidade Católica do Rio de Janeiro, Departamento de Economia.

Inclui bibliografia

1. Finanças - Teses. 2. Aversão ao Risco;. 3. Cassino;. 4. Ciclo Econômico;. 5. Previsão;. 6. Propensão a Apostar;. 7. Mercado de Ações.. I. Monteiro Ribeiro, Ruy. II. Zilberman, Eduardo. III. Pontifícia Universidade Católica do Rio de Janeiro. Departamento de Economia. IV. Título.

CDD: 620.11 


\section{Agradecimentos}

Agradeço aos meus pais pelo apoio incondicional em todas as etapas de minha vida, não sendo esta diferente. Agradeço aos meus orientadores pelo direcionamento da pesquisa. Agradeço também aos membros da banca examinadora pelos valiosos conselhos e sugestões. Por fim, não poderia deixar de agradecer aos amigos pelo companheirismo. 


\section{Resumo}

Cordeiro Alencar, Daniel Lívio; Monteiro Ribeiro, Ruy; Zilberman, Eduardo. Uma proxy para aversão ao risco avaliada no mercado de ações. Rio de Janeiro, 2017. 41p. Dissertação de Mestrado - Departamento de Economia, Pontifícia Universidade Católica do Rio de Janeiro.

Eu calculo uma proxy da propensão à tomada de risco dos jogadores de cassino através dos dados de receita de cassinos. Usando regressões insample e out-of-sample, eu então analiso o quão bem essa proxy prevê o prêmio de risco do mercado de ações.

\section{Palavras-chave}

Aversão ao Risco; Cassino; Ciclo Econômico; Previsão; Propensão a Apostar; Mercado de Ações. 


\section{Abstract}

Cordeiro Alencar, Daniel Lívio; Monteiro Ribeiro, Ruy (Advisor); Zilberman, Eduardo (Co-Advisor). A proxy for risk aversion evaluated in the stock market. Rio de Janeiro, 2017. 41p. Dissertação de Mestrado - Departamento de Economia, Pontifícia Universidade Católica do Rio de Janeiro.

I estimate a proxy for the risk taking behavior of Casino gamblers through a measure of total Casino gambling revenue. Using in-sample and out-of-sample regressions, I then analyze how suited this proxy is in predicting market risk premium.

\section{Keywords}

Risk Aversion; Casino; Business Cycle; Forecast; Propensity to Gamble; Stock Market. 


\section{Sumário}

1 Introdução $\quad 11$

2 Como Estimar a Propensão à Tomada de Risco 13

$\begin{array}{lll}2.1 & \text { Estimação da propensão à tomada de risco } & 14\end{array}$

3 Previsão do prêmio de risco de mercado $\quad 18$

3.1 Previsão in-sample 18

$\begin{array}{lll}3.2 & \text { Previsão out-of-sample } & 24\end{array}$

4 Conclusão $\quad 30$

A Janela temporal da estimação de ptr 34

B Detalhes das variáveis $\quad 35$

$\begin{array}{lll}\text { C Datas de legalização dos cassinos } & 37\end{array}$

D Capacidade preditiva de ptr e ptrc utilizando os modelos apresentados na tabela $2.3 \quad 38$

D.1 DOLS - $0 \quad 38$

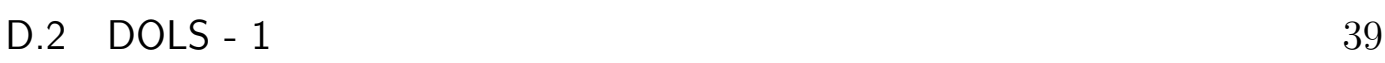

D.3 DOLS - $2 \quad 40$

D.4 DOLS - 3 41 


\section{Lista de figuras}

3.1 Performance de $\widehat{p t r}_{t}$ e $\widehat{\operatorname{ttr}} c_{t}$ na previsão do retorno de seis anos do prêmio de risco do mercado

D.1 Performance de $\widehat{p t r}_{t}$ e $\widehat{p t r}_{t}$ na previsão do retorno de 24 trimestres do prêmio de risco do índice CRSP value-weighted

D.2 Performance de $\widehat{p t r}_{t}$ e $\widehat{p t r}_{t}$ na previsão do retorno de 24 trimestres do prêmio de risco do índice CRSP value-weighted

D.3 Performance de $\widehat{p t r}_{t}$ e $\widehat{p t r} c_{t}$ na previsão do retorno de 24 trimestres do prêmio de risco do índice CRSP value-weighted

D.4 Performance de $\widehat{p t r}_{t}$ e $\widehat{p t r} c_{t}$ na previsão do retorno de 24 trimestres do prêmio de risco do índice CRSP value-weighted 


\section{Lista de tabelas}

2.1 Teste de raiz unitária de Dickey Fuller aumentado 14

2.2 Teste de cointegração de Johansen 15

$\begin{array}{lll}2.3 \text { Modelos } & 16\end{array}$

3.1 Resumo das estatísticas 20

3.2 Regressões in-sample univariadas de previsão do prêmio de risco do mercado 21

3.3 Regressões in-sample multivariadas de previsão do prêmio de risco do mercado 23

3.4 Previsão out-of-sample do prêmio de risco do mercado 25

3.5 Comparação entre $\widehat{p t r}$ e outros previsores 27

3.6 Relação de previsão entre $\widehat{p t r}_{t}$ e as outras variáveis 28

A.1 Teste de quebra estrutural de Quandt-Andrews 34

$\begin{array}{lll}\text { C.1 Legalização dos cassinos } & 37\end{array}$

D.1 Previsão do prêmio de risco do índice CRSP value-weighted 38

D.2 Previsão do prêmio de risco do índice CRSP value-weighted 39

D.3 Previsão do prêmio de risco do índice CRSP value-weighted 40

D.4 Previsão do prêmio de risco do índice CRSP value-weighted 41 


\section{Lista de Abreviaturas}

BEA - Bureau of Economic Analisys

BLS - Bureau of Labor Statistics

CRSP-VW - Center for Research in Security Prices

CV - Custo de Viajar

GAC - Gasto com Apostas em Cassinos

LC - Número de Estados que Legalizaram Cassinos

MQO - Mínimos Quadrados Ordinários

NBER - National Bureau of Economic Research

PIB - Produto Interno Bruno

PTR - Propensão à Tomada de Risco 


\section{Introdução}

Decisões sob risco são frequentes na vida cotidiana. Aspectos emotivos correntes, como estado de humor vigente, alteram a propensão à tomada de risco (1) (2). Nesse estudo, através dos gastos com apostas em cassinos dos Estados Unidos, eu calculo uma proxy para a propensão à tomada de risco dos jogadores de casino ao longo do tempo e avalio a capacidade dessa proxy prever o prêmio de risco do mercado em regressões in-sample e out-of-sample.

Para o cálculo da proxy para a propensão à tomada de risco, eu faço uma regressão dos gastos nos cassinos em controles que têm baixa correlação com o estado de humor dos jogadores e assumo a hipótese de que o resíduo dessa regressão é uma proxy para a propensão à tomada de risco. Analisando essa proxy eu encontro que ela performa melhor que outras variáveis na previsão do prêmio de risco de mercado em regressões out-of-sample. E, além disso, em regressões de previsão in-sample, a proxy pode ser utilizada conjuntamente com outras variáveis para auxiliar na previsão.

Outros trabalhos já estudaram a relação entre $\operatorname{apostas}^{1}$ e investimentos. Por exemplo, (3) relaciona apostas em loteria com decisões de investimento no mercado de ações e decisões corporativas. Considero que ao focar em apostas em cassinos o meu trabalho difere do citado em dois aspectos. Primeiro, pagamento aos vencedores dos jogos de cassinos tem assimetria positiva bem menor que o prêmio recebido pelos vencedores de loterias, ou seja, o prêmio das loterias é bem maior que o custo de apostar e a probabilidade de ganhar na loteria é bem menor que a probabilidade de vencer nos jogos de cassinos. Segundo, Apostas em cassinos diferem de apostas em corridas (4.5 bilhões) porque os jogos de cassinos requerem em geral menos habilidade. Embora alguns jogos de cassinos requeiram certa habilidade, muitos envolvem simplesmente a sorte.

A produção acadêmica para cada tipo de aposta difere tanto em tema como em quantidade de publicações. A literatura sobre loterias é bem vasta, por exemplo, (4) estuda o aumento da receita de loterias em períodos de recessão. Já (5) relaciona loteria com decisões de investimento no mercado

${ }^{1}$ O BEA (Bureau of Economic Analisys) divide a rubrica apostas em três tipos: cassinos, loterias e corridas. Embora esses três tipos de aposta possuam semelhanças, cada tipo possui características próprias. 
de ações. Sobre cassino, há publicações que tentam identificar as variáveis que determinam a demanda por cassino como (6) e (7). Já (8) estuda o efeito de recessões nos gastos com apostas em cassino e encontra que esses gastos aumentam durante períodos de expansão econômica e que choques de renda que não estão ligados ao ciclo econômico não impactam os gastos com apostas em cassinos.

A modelagem teórica do ambiente de apostas é escassa. Uma dificuldade de modelar apostas com funções de utilidade comumente utilizadas é que o payoff esperado é negativo ou próximo de zero. O modelo mais proeminente de apostas em cassinos (9) baseia-se na cumulative prospect theory (10), uma teoria sobre tomada de decisões sob risco que afirma que as pessoas avaliam os risco a partir de uma função valor que é côncava sob ganhos e convexa sob perdas. Nesse modelo três características definem a função utilidade do agente: aversão a perda, concavidade/convexidade e o peso subjetivo dado a eventos de baixa probabilidade. Nesse modelo, com agentes heterogêneos, o humor do agente influencia na decisão de apostar em cassinos da seguinte maneira: quanto menor o parâmetro de aversão a perda, mais tipos de agentes estarão dispostos a entrar em cassinos.

Esse trabalho está estruturado da seguinte maneira: no capítulo 2 eu a construção da proxy para a propensão à tomada de risco. No capítulo $3 \mathrm{eu}$ estudo a sua capacidade preditiva em regressões in-sample e out-of-sample. No capítulo 4 eu concluo. 


\section{2}

\section{Como Estimar a Propensão à Tomada de Risco}

Estimar a aversão ao risco é difícil dado que essa é uma variável latente. Para chegar a estimativas próximas, a literatura utiliza diversas ferramentas. Por exemplo, (11), utiliza dados de corridas de cavalo para identificar o grau de aversão ao risco de diferentes apostadores. (12) propõe a utilização do comportamento da oferta de trabalho. Há ainda trabalhos que calculam uma mudanças na aversão ao risco ao longo do tempo através de surveys que fazem perguntas qualitativas/quantitativas, úteis para casos específicos como, por exemplo, (13) que encontra um aumento na aversão após a crise de 2008.

No entanto, a utilização de surveys impede uma análise temporal sólida e contínua da propensão à tomada de risco dos agentes, dado que os surveys são realizados esporadicamente e com variações substanciais em seus conteúdos. Consequentemente, isso impossibilita a sua utilização como proxy para controlar a aversão ao risco dos agentes em modelos econométricos de séries temporais.

Para substituir a utilização dos surveys, proponho estimar a propensão à tomada de risco dos agentes - ptr - através de gastos com apostas em cassinos. A hipótese por trás da utilização desse dado observado é que, controlado o ambiente econômico do agente, gastos por cassino explicitariam a propensão à tomada de risco do agente em questão.

Para estimar esse efeito, proponho o seguinte modelo econométrico.

$$
\text { Gastos em } \operatorname{cassinos}_{t}=\alpha[\text { ambiente econômico }]_{t}+\epsilon_{t}
$$

Os componentes do vetor ambiente econômico tentam controlar para todos os fatores que levariam o agente a gastar com apostas em cassinos, mas não tem a ver com a aversão ao risco intrínseca do agente. Utilizo o PIB para controlar para choques de renda em geral. Para controlar custos de transação e dificuldade de se conseguir apostar em cassinos, utilizo a variação na quantidade de estados que legalizaram atividades de cassinos e também o custo médio de deslocamento. O detalhamento dessas variáveis encontra-se no apêndice.

O interesse principal de estimar a regressão dessa maneira é recuperar tudo o que explica gastos em cassinos que não são explicados pelo ambiente 
econômico do agente, ou seja, recuperar somente os fatores não observáveis que afetam gastos em cassinos apenas através da sua propensão à tomada de risco.

A equação a ser estimada fica então da seguinte maneira:

$$
g a c_{t}=\alpha+\beta_{p i b} p i b_{t}+\beta_{c v} c v_{t}+\beta_{L C} L C_{t}+\epsilon_{t}
$$

$\mathrm{Na}$ regressão acima gac é o logaritmo do gasto real com apostas em cassino, pib é o logaritmo do produto interno bruto real, LC é o número de estados que legalizaram cassinos. Estimados os parâmetros, o resíduo da regressão acima, $\epsilon_{t}$ me trará informação sobre a propensão média a tomada de risco do agente (ptr).

\section{1}

\section{Estimação da propensão à tomada de risco}

A princípio a estimação direta dos parâmetro da regressão por OLS pode parecer ingênua, dado um potencial problema de endogeneidade. Porém, como exposto por (14), se as variáveis tiverem uma relação de cointegração, os parâmetros serão super consistentes, eliminando o problema de endogeneidade da estimação.

Tabela 2.1: Teste de raiz unitária de Dickey Fuller aumentado

\begin{tabular}{lrrrr}
\hline & \multicolumn{2}{c}{ nível } & \multicolumn{2}{c}{ primeira diferença } \\
\cline { 2 - 5 } Variável & estatística t & p-valor & estatística t & p-valor \\
\hline$g a c_{t}$ & -1.57 & 0.49 & -4.43 & 0.00 \\
pib $_{t}$ & -1.52 & 0.52 & -4.86 & 0.00 \\
$c v_{t}$ & -2.31 & 0.17 & -10.15 & 0.00 \\
$L C_{t}$ & -1.26 & 0.64 & -9.01 & 0.00 \\
\hline
\end{tabular}

Nota: Para o período de 1981T1-2016T4, quando avaliadas em nível, nenhum variável rejeitou a hipótese nula de possuir raiz unitária para ao nível de significância de 1\%. Quando avaliadas as primeiras diferenças, todas as variáveis rejeitaram a hipótese nula de possuir raiz unitária ao nível de significância de $1 \%$.

Para avaliar a existência de cointegração, primeiramente eu testei se as variáveis passavam no teste de raiz unitária de Dickey-Fuller aumentado. Os resultados ${ }^{1}$ estão apresentados na tabela 2.1. Dado que nenhuma variável rejeitou a hipótese nula de possuir raiz unitária, eu prossegui para o teste de cointegração de Johansen. Esse teste avalia a hipótese H0 de haver r relações de cointegração contra a hipótese de haver $\mathrm{H} 1=\mathrm{r}+1$ relações de cointegração.

${ }^{1}$ Para os testes apresentados e subsequentes estimações, eu utilizei dados trimestrais do primeiro trimestre de 1981 até o quarto trimestre de 2016. Nos anexos, estão os detalhes para a escolha dessa data. 
Os resultados estão apresentados na tabela 2.2. As duas estatísticas do teste de Johansen, o autovalor máximo e o teste do traço, apresentaramm fortes evidências de haver uma única relação de cointegração entre $g a c_{t}, L C_{t}, p_{i} b_{t} \mathrm{e}$ $c v_{t}$.

Tabela 2.2: Teste de cointegração de Johansen

\begin{tabular}{|c|c|c|c|c|}
\hline \multicolumn{2}{|c|}{ Autovalor Máximo } & \multicolumn{2}{|l|}{ Traço } & \multirow[t]{2}{*}{$\mathrm{H} 0=\mathrm{r}$} \\
\hline Estatística teste & $90 \% \mathrm{VC}$ & Estatística teste & $90 \% \mathrm{VC}$ & \\
\hline \multicolumn{5}{|c|}{ Painel A - intervalo do lag: 0 a 1} \\
\hline 25.34 & 25.12 & 49.00 & 44.49 & 0 \\
\hline 15.71 & 18.89 & 23.66 & 27.07 & 1 \\
\hline 5.15 & 12.30 & 7.95 & 13.43 & 2 \\
\hline 2.80 & 2.71 & 2.80 & 2.71 & 3 \\
\hline \multicolumn{5}{|c|}{ Painel A - intervalo do lag: 1 a 2} \\
\hline 25.34 & 25.12 & 45.35 & 44.49 & 0 \\
\hline 12.12 & 18.89 & 20.01 & 27.07 & 1 \\
\hline 5.65 & 12.30 & 7.89 & 13.43 & 2 \\
\hline 2.24 & 2.71 & 2.24 & 2.71 & 3 \\
\hline \multicolumn{5}{|c|}{ Painel A - intervalo do lag: 1 a 3} \\
\hline 31.21 & 25.12 & 52.21 & 44.49 & 0 \\
\hline 13.43 & 18.89 & 21.00 & 27.07 & 1 \\
\hline 5.47 & 12.30 & 7.57 & 13.43 & 2 \\
\hline 2.10 & 2.71 & 2.10 & 2.71 & 3 \\
\hline \multicolumn{5}{|c|}{ Painel A - intervalo do lag: 1 a 4} \\
\hline 26.43 & 25.12 & 51.55 & 44.49 & 0 \\
\hline 16.67 & 18.89 & 25.12 & 27.07 & 1 \\
\hline 5.47 & 12.30 & 8.45 & 13.43 & 2 \\
\hline 2.98 & 2.71 & 2.98 & 2.71 & 3 \\
\hline
\end{tabular}

Nota: Os resultados apresentados nessa tabela não assumem tendência linear nos dados, mas assume que a relação de cointegração tem uma constante (15). O teste de Johansen testa a hipótese HO de haver $r$ relações de cointegração contra a hipótese de haver $H 1=r+1$ relações de cointegraçao. A primeira coluna estatística teste informa o valor da estatítica de teste para o teste do autovalor máximo e a segunda informa o valor da estatística de teste para o teste do traço. 90\% VC informa o valor da estatística teste para o intervalo de confiança de 90\%. Ambos os tetes apresentam forte evidências de haver uma única relação de cointegração entre gac $c_{t}, L C_{t}$, pib $b_{t}$ e $c v_{t}$ intedependente da especificação do número de lags. 
$g a c_{t}=\alpha+\beta_{p i b} p i b_{t}+\beta_{c v} c v_{t}+\beta_{L C} L C_{t}+\sum_{i=-k}^{i=k} b_{p i b, i} \Delta p i b_{t-i}+\sum_{i=-k}^{i=k} b_{c v, i} \Delta c v_{t-i}+e_{t}$

Dado que as variáveis cointegram, para o cálculo da $\widehat{p t r}_{t}$ e das estimativas dos coeficientes da regressão eu utilizei a regressão OLS (Ordinary Least Squares) e também, seguindo (14), utilizei DOLS (Dynamic OLS), que foi proposta em (16). Essa metodologia gera estimativas consistentes dos coeficientes de cointegração em um ambiente multivariado. A estimação por DOLS consiste em adicionar como regressores lags e leads da primeira diferença das variáveis independentes ${ }^{2}$, conforme equação 2-3, em seguida é feita uma regressão de mínimos quadrados ordinários.

Tabela 2.3: Modelos

\begin{tabular}{cccccc}
\hline Modelo & lags\&leads & constant & pib & cv & LC \\
\hline OLS & - & $-7,48$ & 1,28 & $-0,44$ & 0,07 \\
DLS & 0 & $-6,93$ & 1,23 & $-0,45$ & 0,07 \\
DLS & 1 & $-6,16$ & 1,18 & $-0,51$ & 0,07 \\
DLS & 2 & $-5,17$ & 1,11 & $-0,58$ & 0,07 \\
DLS & 3 & $-3,84$ & 1,02 & $-0,67$ & 0,07 \\
\hline
\end{tabular}

Nota: A primeira linha apresenta os coeficientes da regressão $O L S$ e as linhas seguintes apresentam os parâmetros obtidos por DOLS ("Dynamic OLS"), seguindo (16), com lags e leads das variáveis independentes. Não foram colocados "lags"e "leads"da variável $L C$, porque essa variável tem pouca variação. A primeira diferença desse vetor é uma sequência de 216 zeros e 16 uns, assim se forem adicionados vários lags e leads dessa variável, haverá um problema de multicolinearidade

Foram testadas quatro variações da equação 2-3, com diferentes quantidades de lags e leads, o coeficiente dessas regressões são apresentados na tabela 2.3. Tanto os coeficientes da regressão quanto os resultados gerais apresentados a seguir não apresentam grandes variações a escolha do modelo. Por simplicidade no restante deste trabalho irei apresentar apenas os resultados da regressão OLS.

A equação 2-4 reporta os parâmetros da regressão OLS e a estatística t com os erros padrão corridos pelo método de Newey-West. A regressão foi feita utilizando dados do primeiro trimestre de 1981 até o quarto trimestre de 2016. A estimativa para o coeficiente do pib é de 1,28 , indicando uma relação positiva entra gastos em cassino e PIB. O coeficiente da variável cv é de -0,43, indicando que quando o custo de viajar é baixo, os gastos em jogos de cassino

\footnotetext{
${ }^{2}$ Não foram colocados "lags"e "leads"da variável LC, porque essa variável tem pouca variação. A primeira diferença desse vetor é uma sequência de 216 zeros e 16 uns, assim se forem adicionados vários lags e leads dessa variável, haverá um problema de multicolinearidade
} 
são maiores. O coeficiente positivo da variável LC indica que quando mais estados legalizam cassinos, há mais gastos com apostas em cassinos.

$$
\begin{array}{r}
\text { gac }_{t}=-7,48 \\
(-2,53)
\end{array} \quad+\begin{aligned}
& 1,28 p i b_{t} \\
& (5,12)
\end{aligned} \quad \begin{gathered}
\left(-2,73 c v_{t}\right. \\
\end{gathered}
$$




\section{3 \\ Previsão do prêmio de risco de mercado}

Assumindo que $\widehat{p t r}_{t}$, estimado como resíduo da regressão 2-2 aproxima bem o coeficiente de propensão à tomada de risco médio dos apostadores, eu testo agora sua capacidade de prever o prêmio de risco do mercado. Para tal, faço comparações da capacidade preditiva de $\widehat{p t r}_{t}$ e demais variáveis utilizadas na literatura, como $\widehat{c a y}_{t}$, Dividend Price Ratio, Investment to Capital Ratio, detalhadas a seguir. Os testes seguirão os execícios propostos em (17), (14) e (18).

\section{1}

\section{Previsão in-sample}

A típica análise de previsão in-sample é feita a partir de regressões OLS, regredindo o prêmio de risco em lags dos previsores, conforme equação 3-1.

$$
\text { prêmiot }_{t, t+h}=\alpha+\beta X_{t}+e_{t, t+h}
$$

O prêmio prtth $_{t+h}$ é composição contínua do excesso de retorno de "h" trimestres, $r_{t+1}-r_{f, t+1}+\ldots+r_{t+h}-r_{f, t+h}$. Sendo $\mathbf{r}_{\mathbf{t}}$ o logaritmo natural do índice CRSP-VW ${ }^{1} ; \mathbf{r}_{\mathbf{f}, \mathbf{t}}$ é o logaritmo natural do retorno do 3-month treasury bill, negociado no mercado secundário no último dia do mês t-1. " $X_{t}$ " é o vetor de previsão, que pode ser unidimensional ou multidimensional, dependendo do número de previsores utilizados na regressão.

Os previsores em estudo, nesse trabalho, são classificadas em dois grupos. O primeiro grupo é composto por variáveis que são desvios de uma tendência. São elas: $\widehat{\mathbf{p t r}}_{\mathbf{t}}$, construída conforme descrito na seção anterior; $\widehat{\mathbf{c a y}}_{\mathbf{t}}$ - Consumption-Wealth Ratio ${ }^{2}$, proposto em (14) - expresso como: $\widehat{c}_{t}=$ $c_{t} \widehat{\alpha}-\widehat{\beta}_{a} a_{t}-\widehat{\beta_{y}} y_{t}$, onde $c_{t}$ é o consumo agregado, $y_{t}$ é a renda agregada do trabalho e $a_{t}$ é a riqueza das famílias. Os coeficientes $\widehat{\alpha}, \widehat{\beta}_{a}$ e $\widehat{\beta}_{y}$ são estimados por Dynamic OLS $\left(\mathrm{DOLS}^{3}\right)$, regredindo $c_{t}$ em $a_{t}$ e $y_{t}$.

${ }^{1} \mathrm{O}$ índice CRSP-VW é o retorno de uma carteira composta por todas as ações do mercado acionário americano ponderadas pelo valor.

${ }^{2} \mathrm{Os}$ dados para a construção dessa série foram colhidos do site http://faculty.haas.berkeley.edu/lettau/data_cay.html

${ }^{3}$ Nessa regressão foram utilizados 8 lags e 8 leads, conforme (14) 
O segundo grupo de previsores é composto por variáveis que guardam relação ou com a taxa de juros ou com as características das ações. São elas: $\mathbf{d}_{\mathbf{t}} / \mathbf{p}_{\mathbf{t}}$ - Dividend Price Ratio, é a razão $d_{t} / p_{t}$, onde $d_{t}$ é a soma dos dividendos dos últimos 12 meses do índice CRSP-VW $\left(d_{t}+\ldots+d_{t-11}\right)$ e $p_{t}$ é o índice CRSP-VW; ik - Investment to Capital Ratio, proposta em (19) - é a razão do investimento agregado sobre o capital agregado de toda a economia ${ }^{4}$; bm - book value, explorada por exemplo em (20) e (21) - é o valor contábil sobre o valor de mercado do Dow Jones Industrial Average; tbl - treasury bills, estudada por exemplo em (22) e (23) - é a taxa de juros livre de risco ; lty - Long Term Yield, avaliada por exemplo em (23) e (24) - é o rendimento de títulos públicos de longo prazo; tms - term spread, explorada por exemplo em (22) e (23) - é a diferença entre o rendimento de títulos públicos de longo prazo e a taxa de juros livre de risco; dfy - Default Yield Spread, estudada por exemplo em (25) e (24) - é a diferença entre o rendimento de títulos corporativos classificados como BAA e o rendimento de títulos corporativos classificados como AAA; infl - inflation, avaliada por exemplo (26), (27) e (28) - é a taxa de inflação dos Estados Unidos.

Uma vez que as propriedades dos previsores propostos por outros autores já foram amplamente discutidas em publicações anteriores, adiante irei discutir unicamente os resultados referentes a $\widehat{p t r}_{t}$.

O painel A da tabela 3.1, construída utilizando dados do período 1981Q12015Q4, apresenta a média, o desvio padrão e a autocorrelação com lags de um a vinte trimestres. O desvio padrão de $\widehat{p t r}_{t}$ é o que mais se aproxima do desvio padrão do prêmio de risco do mercado. A autocorrelação de $\widehat{p t r}_{t}$ é relativamente baixa e varia de 0,88 (lag de um trimestre) a -0,20 (lag de 20 trimestres).

O painel B da tabela 3.1 apresenta os coeficientes de correlação. No período em análise, a variável que apresentou maior correlação em módulo com $\widehat{p t r}_{t}$ foi ik $(-0,56)$.

\footnotetext{
${ }^{4} d_{t} / p_{t}$ foi construída a partir de dados da série CRSP-VW. Todas as outras séries desse grupo foram colhidas do site http://www.hec.unil.ch/agoyal/
} 
Tabela 3.1: Resumo das estatísticas

\begin{tabular}{|c|c|c|c|c|c|c|c|c|c|c|c|c|}
\hline & $\begin{array}{c}\text { Lags de } \\
\text { autocorrelações } \\
\text { (em trimestres) }\end{array}$ & $r_{t}-r_{f}$ & $\widehat{p t r}$ & $\widehat{c a y}$ & $\mathrm{~d} / \mathrm{p}$ & $\mathrm{ik}$ & $\mathrm{bm}$ & tbl & lty & tms & dfy & infl \\
\hline Média & & 0,01 & 0,00 & 0,01 & $-3,75$ & 0,04 & 0,40 & 0,04 & 0,07 & 0,02 & 0,01 & 0,01 \\
\hline desvio padrão & & 0,09 & 0,08 & 0,02 & 0,39 & 0,00 & 0,22 & 0,03 & 0,03 & 0,01 & 0,00 & 0,01 \\
\hline Autocorrelações & 1 & 0,05 & 0,88 & 0,87 & 0,97 & 0,98 & 0,96 & 0,95 & 0,96 & 0,96 & 0,83 & 0,18 \\
\hline . & 2 & $-0,02$ & 0,78 & 0,80 & 0,93 & 0,92 & 0,91 & 0,88 & 0,91 & 0,91 & 0,65 & 0,09 \\
\hline . & 3 & $-0,05$ & 0,69 & 0,73 & 0,89 & 0,85 & 0,85 & 0,82 & 0,87 & 0,87 & 0,55 & 0,18 \\
\hline r. & 4 & $-0,04$ & 0,56 & 0,64 & 0,85 & 0,77 & 0,79 & 0,76 & 0,82 & 0,82 & 0,45 & 0,05 \\
\hline & 5 & $-0,01$ & 0,46 & 0,57 & 0,82 & 0,68 & 0,73 & 0,70 & 0,78 & 0,78 & 0,38 & 0,12 \\
\hline & 6 & $-0,01$ & 0,37 & 0,51 & 0,78 & 0,60 & 0,67 & 0,64 & 0,74 & 0,74 & 0,35 & 0,11 \\
\hline & 7 & $-0,12$ & 0,27 & 0,45 & 0,75 & 0,53 & 0,62 & 0,60 & 0,72 & 0,72 & 0,27 & 0,04 \\
\hline & 8 & 0,02 & 0,20 & 0,38 & 0,72 & 0,46 & 0,59 & 0,57 & 0,71 & 0,71 & 0,22 & 0,07 \\
\hline & 9 & $-0,04$ & 0,12 & 0,34 & 0,70 & 0,41 & 0,57 & 0,54 & 0,70 & 0,70 & 0,18 & 0,01 \\
\hline & 10 & 0,07 & 0,04 & 0,29 & 0,69 & 0,35 & 0,55 & 0,51 & 0,69 & 0,69 & 0,18 & 0,05 \\
\hline & 11 & 0,01 & 0,00 & 0,26 & 0,66 & 0,31 & 0,53 & 0,48 & 0,67 & 0,67 & 0,22 & 0,10 \\
\hline & 12 & $-0,03$ & $-0,05$ & 0,24 & 0,64 & 0,27 & 0,50 & 0,46 & 0,64 & 0,64 & 0,23 & 0,16 \\
\hline & 13 & $-0,13$ & $-0,09$ & 0,21 & 0,61 & 0,23 & 0,47 & 0,42 & 0,61 & 0,61 & 0,24 & $-0,06$ \\
\hline & 14 & 0,00 & $-0,10$ & 0,18 & 0,59 & 0,19 & 0,43 & 0,40 & 0,57 & 0,57 & 0,23 & 0,19 \\
\hline & 15 & 0,00 & $-0,12$ & 0,18 & 0,57 & 0,15 & 0,40 & 0,37 & 0,53 & 0,53 & 0,16 & $-0,06$ \\
\hline & 16 & 0,06 & $-0,16$ & 0,20 & 0,54 & 0,12 & 0,37 & 0,36 & 0,50 & 0,50 & 0,12 & $-0,02$ \\
\hline & 17 & $-0,04$ & $-0,17$ & 0,20 & 0,50 & 0,08 & 0,33 & 0,35 & 0,47 & 0,47 & 0,10 & 0,09 \\
\hline & 18 & $-0,03$ & $-0,18$ & 0,22 & 0,47 & 0,04 & 0,30 & 0,35 & 0,44 & 0,44 & 0,06 & $-0,04$ \\
\hline & 19 & $-0,15$ & $-0,20$ & 0,23 & 0,43 & 0,00 & 0,27 & 0,34 & 0,42 & 0,42 & 0,05 & 0,02 \\
\hline & 20 & $-0,16$ & $-0,20$ & 0,25 & 0,40 & $-0,03$ & 0,25 & 0,34 & 0,41 & 0,41 & 0,04 & 0,01 \\
\hline \multicolumn{13}{|c|}{ Painel B: correlação } \\
\hline & & $r_{t}-r_{f}$ & $\widehat{c a y}$ & $\mathrm{~d} / \mathrm{p}$ & $\mathrm{ik}$ & $\mathrm{bm}$ & tbl & lty & tms & dfy & infl & \\
\hline & $\widehat{p t r}$ & 0,03 & $-0,07$ & 0,17 & $-0,56$ & 0,04 & $-0,25$ & $-0,11$ & 0,43 & $-0,03$ & $-0,03$ & \\
\hline & $r_{t}-r_{f}$ & & $-0,10$ & $-0,11$ & $-0,10$ & $-0,09$ & $-0,08$ & $-0,06$ & 0,07 & $-0,18$ & 0,00 & \\
\hline & $\widehat{c a y}$ & & & 0,31 & 0,18 & 0,09 & 0,41 & 0,50 & 0,07 & $-0,01$ & 0,12 & \\
\hline & $\mathrm{d} / \mathrm{p}$ & & & & $-0,36$ & 0,87 & 0,59 & 0,71 & 0,08 & 0,57 & 0,28 & \\
\hline & ik & & & & & $-0,18$ & 0,42 & 0,23 & $-0,58$ & $-0,21$ & 0,11 & \\
\hline & $\mathrm{bm}$ & & & & & & 0,67 & 0,76 & 0,00 & 0,66 & 0,34 & \\
\hline & tbl & & & & & & & 0,93 & $-0,51$ & 0,24 & 0,49 & \\
\hline & lty & & & & & & & & $-0,14$ & 0,32 & 0,46 & \\
\hline & tms & & & & & & & & & 0,09 & $-0,24$ & \\
\hline & dfy & & & & & & & & & & $-0,08$ & \\
\hline
\end{tabular}

Nota: No painel A, é reportada a média, o desvio padrão e a autocorrelação com lags de 1 a 20 das variáveis utilizadas na regressão de previsão. $\mathrm{O}$ painel $\mathrm{B}$ apresenta a correlação entre as variáveis. $\mathbf{r}_{\mathbf{t}}-\mathbf{r}_{\mathbf{f}, \mathbf{t}}$ é o prêmio de risco do mercado, onde $\mathbf{r}_{\mathbf{t}}$ é o logaritmo natural do índice CRSP e $\mathbf{r}_{\mathbf{f}, \mathbf{t}}$ é o retorno do 3-month treasury bill; $\widehat{\mathbf{p t r}}_{\mathbf{t}}$ é a propensão a tomada de risco; $\widehat{\text { cay }}_{\mathbf{t}}-$ Consumption-Wealth Ratio - é $\widehat{c a y}_{t}=c_{t} \widehat{\alpha}-\widehat{\beta}_{a} a_{t}-\widehat{\beta_{y}} y_{t}$, onde $c_{t}$ é o consumo agregado, $y_{t}$ é a renda agregada do trabalho e $a_{t}$ é a riqueza das famílias; $\mathbf{d}_{\mathbf{t}} / \mathbf{p}_{\mathbf{t}}$ - Dividend Price Ratio - é o logaritmo da razão $d_{t} / p_{t}$, onde $d_{t}$ é a soma dos dividendos dos últimos 12 meses do índice CRSP $\left(d_{t}+\ldots+d_{t-11}\right)$ e $p_{t}$ é o valor do índice CRSP; ik - Investment to Capital Ratio - é a razão do investimento agregado sobre o capital agregado de toda a economia; bm - book value - é o valor contábil sobre o valor de mercado do Dow Jones Industrial Average; tbl trasury bills - é a taxa de juros livre de risco ; lty - Long Term Yield - é o rendimento de títulos públicos de longo prazo; tms - term spread - é a diferença entre o rendimento de títulos públicos de longo prazo e a taxa de juros livre de risco; dfy - Default Yield Spread - é a diferença entre o rendimento de títulos corporativos classificados como BAA e o rendimento de títulos corporativos classificados como AAA; infl - inflation - é a taxa de inflação dos Estados Unidos. O período amostral para o cálculo dessas estatísticas é $1981 \mathrm{~T} 1$ $2015 \mathrm{~T} 4$. 
Na tabela 3.2, são apresentadas estatísticas de previsão calculadas utilizando a forma univariada da equação 3-1, com a estatística-t corrigida pelo método de Newey-West (29). Esse correção é necessária devida à correlação dos resíduos proporcionada pelo overlap dos períodos. A primeira coluna mostra que o coeficiente de $\widehat{p t r}_{t}$ é sempre positivo. Esse resultado reforça a ideia de que quanto mais propensos os agentes estão a tomada de risco, maior a demanda por risco e, portanto, menor é o prêmio de risco.

A tabela 3.2 também mostra que a capacidade preditiva de $\widehat{p t r}_{t}$ aumenta com o aumento do horizonte de previsão. Duas estatísticas reforçam essa relação. A primeira, em colchetes, é o $\bar{R}^{2}$ (R-quadrado ajustado), o qual se torna maior conforme o horizonte de previsão aumenta. A segunda, em parenteses, é a estatística t corrigida, que é significante ao nível de $5 \%$ apenas quando o horizonte de previsão é longo (4, 5 e 6 anos). Esse crescimento das estatísticas com o horizonte estão em linha com a literatura de previsão de retorno de longo prazo (veja, por exemplo, (30) e (31)).

Tabela 3.2: Regressões in-sample univariadas de previsão do prêmio de risco do mercado

Período de previsão $1981 \mathrm{~T} 1$ - 2015T4

\begin{tabular}{|c|c|c|c|c|c|c|c|c|c|c|}
\hline & $\widehat{p t r}_{t}$ & $\widehat{\operatorname{cay}}_{t}$ & $\mathrm{bm}$ & dfy & $\mathrm{d} / \mathrm{p}$ & ifl & $\mathrm{ik}$ & lty & tbl & tms \\
\hline \multicolumn{11}{|c|}{ Previsão de 4 trimestres } \\
\hline Coeficiente & 0,31 & 0,96 & 0,09 & 0,87 & 0,06 & $-4,95$ & $-10,75$ & $-0,20$ & $-0,53$ & 2,45 \\
\hline estatística-t & $(0,99)$ & $(0,93)$ & $(0,62)$ & $(0,17)$ & $(0,87)$ & $(-1,60)$ & $(-1,58)$ & $(-0,23)$ & $(-0,71)$ & $(1,34)$ \\
\hline $\bar{R}^{2}(\%)$ & {$[0,01]$} & {$[0,00]$} & {$[0,00]$} & {$[-0,01]$} & {$[0,01]$} & {$[0,03]$} & {$[0,05]$} & {$[-0,01]$} & {$[0,00]$} & {$[0,03]$} \\
\hline \multicolumn{11}{|c|}{ Previsão de 8 trimestres } \\
\hline & 0,70 & 3,74 & 0,23 & 5,66 & 0,17 & $-2,99$ & $-21,46$ & 0,29 & $-0,79$ & 6,24 \\
\hline & $(1,20)$ & $(1,53)$ & $(1,22)$ & $(0,94)$ & $(1,45)$ & $(-1,44)$ & $(-1,61)$ & $(0,25)$ & $(-1,04)$ & $(2,28)$ \\
\hline & {$[0,05]$} & {$[0,06]$} & {$[0,04]$} & {$[0,01]$} & {$[0,08]$} & {$[-0,00]$} & {$[0,12]$} & {$[-0,01]$} & {$[0,00]$} & {$[0,12]$} \\
\hline \multicolumn{11}{|c|}{ Previsão de 12 trimestres } \\
\hline & 1,01 & 6,92 & 0,23 & 5,31 & 0,23 & $-2,57$ & $-36,10$ & 0,37 & $-1,44$ & 10,24 \\
\hline & $(1,48)$ & $(2,22)$ & $(0,97)$ & $(0,61)$ & $(1,57)$ & $(-1,15)$ & $(-2,60)$ & $(0,24)$ & $(-1,44)$ & $(3,16)$ \\
\hline & {$[0,07]$} & {$[0,15]$} & {$[0,03]$} & {$[0,00]$} & {$[0,11]$} & {$[-0,00]$} & {$[0,24]$} & {$[-0,01]$} & {$[0,02]$} & {$[0,23]$} \\
\hline \multicolumn{11}{|c|}{ Previsão de 16 trimestres } \\
\hline & 1,29 & 7,52 & 0,34 & 10,60 & 0,30 & $-2,71$ & $-47,85$ & 1,33 & $-1,12$ & 11,82 \\
\hline & $(2,34)$ & $(2,20)$ & $(1,55)$ & $(1,07)$ & $(2,34)$ & $(-1,23)$ & $(-4,86)$ & $(0,75)$ & $(-0,85)$ & $(3,95)$ \\
\hline & {$[0,10]$} & {$[0,14]$} & {$[0,06]$} & {$[0,02]$} & {$[0,16]$} & {$[-0,00]$} & {$[0,36]$} & {$[0,01]$} & {$[0,01]$} & {$[0,27]$} \\
\hline \multicolumn{11}{|c|}{ Previsão de 20 trimestres } \\
\hline & 1,79 & 6,57 & 0,47 & 18,66 & 0,39 & $-1,21$ & $-54,15$ & 2,34 & $-0,43$ & 11,40 \\
\hline & $(3,66)$ & $(1,77)$ & $(2,40)$ & $(1,70)$ & $(3,76)$ & $(-0,34)$ & $(-7,86)$ & $(1,22)$ & $(-0,25)$ & $(3,11)$ \\
\hline & {$[0,18]$} & {$[0,09]$} & {$[0,11]$} & {$[0,07]$} & {$[0,25]$} & {$[-0,01]$} & {$[0,40]$} & {$[0,03]$} & {$[-0,01]$} & {$[0,22]$} \\
\hline \multicolumn{11}{|c|}{ Previsão de 24 trimestres } \\
\hline & 1,77 & 4,38 & 0,47 & 18,22 & 0,42 & 1,46 & $-56,78$ & 2,89 & 0,25 & 9,96 \\
\hline & $(4,09)$ & $(1,29)$ & $(2,46)$ & $(1,79)$ & $(4,74)$ & $(0,35)$ & $(-7,98)$ & $(1,62)$ & $(0,13)$ & $(2,31)$ \\
\hline & {$[0,22]$} & {$[0,04]$} & {$[0,13]$} & {$[0,09]$} & {$[0,35]$} & {$[-0,01]$} & {$[0,47]$} & {$[0,06]$} & {$[-0,01]$} & {$[0,20]$} \\
\hline
\end{tabular}

Nota: A tabela apresenta estatísticas de regressões in-sample univariadas de previsão do prêmio de risco do mercado. As estão variáveis estão apresentadas na tabela 3.1. Para cada regressão a tabela reporta o corficiente de cada previsor, a estatística-t (entre parênteses)com a correção proposta em (29) e o R quadrado ajustado (entre colchetes). A amostra é trimestral do período 1981T1-2015T4. 
Embora a elevada persistência das variáveis e os elevados valores das estatísticas t e $\bar{R}^{2}$ geralmente encontrados na literatura de previsão do retorno de longos horizontes possam refletir um problema de regressão espúria (veja, por exemplo, (32)), é importante ressaltar que no presente trabalho poucas variáveis apresentaram estatísticas elevadas. Apenas 50\% apresentaram uma estatística t-corrigida significante. Apenas $\widehat{p t r}_{t}$ e outras duas variáveis apresentaram $\bar{R}^{2}$ maior que $20 \%$.

Dentre essas três variáveis, $\widehat{p t r}_{t}$ foi a que apresentou menor persistência. Esse resultado, juntamente com o sucesso na previsão out-of-sample utilizando $p t r_{t}$, resultado a ser apresentado na próxima seção, e o fato de a autocorrelação da variável dependente ser próxima de zero. Fazem com que eu possa afirmar que a preocupação econométrica em estimativas de previsibilidade, o risco de uma regressão espúria, está relativamente mitigados quando $p t r_{t}$ é utilizado como variável previsora. (Veja, por exemplo, (33)) e (14)).

Na tabela 3.3, são apresentadas estatísticas de previsão calculadas utilizando versões multivariada da equação 3-1. Para construção das versões multivariadas, $\widehat{p t r}_{t}$ foi incluído nas regressões univariadas que apresentaram coeficiente significante a $5 \%$. Na maioria dos casos, a inclusão de $\widehat{p t r}_{t}$ aumentou o $\bar{R}^{2}$ e a estatística-t de $\widehat{p t r}_{t}$ foi significante. A única exceção ocorreu quando $\widehat{p t r}_{t}$ foi incluído na regressão que utilizava unicamente $i k_{t}$ como previsor. Nessa regressão, a estatística-t de $\widehat{p t r}_{t}$ não foi significante e o $\bar{R}^{2}$ não aumentou em todos os horizontes de previsão. Tal resultado não surpreende dada a elevada correlação entre $\widehat{p t r}_{t}$ e $i k_{t}$ apresentada na tabela 3.1. 
Tabela 3.3: Regressões in-sample multivariadas de previsão do prêmio de risco do mercado

\begin{tabular}{|c|c|c|c|c|c|c|c|}
\hline \multicolumn{8}{|c|}{ Período de previsão $1981 \mathrm{~T} 1$ - 2015T4 } \\
\hline \multicolumn{8}{|c|}{ Horizonte de previsão em tirmestres } \\
\hline Modelo & Regressores & 4 & 8 & 12 & 16 & 20 & 24 \\
\hline \multicolumn{8}{|c|}{ Excesso de retorno de mercado } \\
\hline 1 & $\widehat{p t r}_{t}$ & $\begin{array}{c}0.32 \\
(1.03)\end{array}$ & $\begin{array}{c}0.64 \\
(1.36)\end{array}$ & $\begin{array}{c}0.87 \\
(1.66)\end{array}$ & $\begin{array}{c}1.11 \\
(2.47)\end{array}$ & $\begin{array}{c}1.64 \\
(3.70)\end{array}$ & $\begin{array}{c}1.67 \\
(3.67)\end{array}$ \\
\hline & $\widehat{c a y}_{t}$ & $\begin{array}{c}2.32 \\
(1.95) \\
{[0.06]}\end{array}$ & $\begin{array}{l}5.48 \\
(2.35) \\
{[0.19]}\end{array}$ & $\begin{array}{l}7.63 \\
(2.73) \\
{[0.25]}\end{array}$ & $\begin{array}{c}7.36 \\
(2.34) \\
{[0.24]}\end{array}$ & $\begin{array}{c}5.53 \\
(1.71) \\
{[0.25]}\end{array}$ & $\begin{array}{c}3.16 \\
(1.14) \\
{[0.23]}\end{array}$ \\
\hline 2 & $\widehat{p t r}_{t}$ & $\begin{array}{c}0.23 \\
(0.73)\end{array}$ & $\begin{array}{c}0.51 \\
(0.99)\end{array}$ & $\begin{array}{c}0.76 \\
(1.32)\end{array}$ & $\begin{array}{c}0.97 \\
(2.16)\end{array}$ & $\begin{array}{c}1.38 \\
(3.62)\end{array}$ & $\begin{array}{c}1.32 \\
(4.33)\end{array}$ \\
\hline & $\mathrm{d} / \mathrm{p}$ & $\begin{array}{l}0.10 \\
(1.43) \\
{[0.05]}\end{array}$ & $\begin{array}{c}0.19 \\
(1.78) \\
{[0.14]}\end{array}$ & $\begin{array}{c}0.24 \\
(1.98) \\
{[0.18]}\end{array}$ & $\begin{array}{c}0.30 \\
(2.85) \\
{[0.26]}\end{array}$ & $\begin{array}{c}0.37 \\
(4.74) \\
{[0.40]}\end{array}$ & $\begin{array}{c}0.38 \\
(6.09) \\
{[0.49]}\end{array}$ \\
\hline 3 & $\widehat{p t r}_{t}$ & $\begin{array}{c}0.09 \\
(0.29)\end{array}$ & $\begin{array}{c}0.23 \\
(0.52)\end{array}$ & $\begin{array}{c}0.32 \\
(0.65)\end{array}$ & $\begin{array}{c}0.56 \\
(1.52)\end{array}$ & $\begin{array}{c}1.25 \\
(2.70)\end{array}$ & $\begin{array}{c}1.42 \\
(3.00)\end{array}$ \\
\hline & $\operatorname{tms}$ & $\begin{array}{c}3.00 \\
(1.76) \\
{[0.05]}\end{array}$ & $\begin{array}{l}6.31 \\
(2.60) \\
{[0.15]}\end{array}$ & $\begin{array}{c}9.40 \\
(4.08) \\
{[0.24]}\end{array}$ & $\begin{array}{c}9.85 \\
(3.73) \\
{[0.26]}\end{array}$ & $\begin{array}{c}7.37 \\
(1.93) \\
{[0.26]}\end{array}$ & $\begin{array}{c}5.09 \\
(1.18) \\
{[0.25]}\end{array}$ \\
\hline 4 & $\widehat{p t r}_{t}$ & $\begin{array}{c}0.02 \\
(0.05)\end{array}$ & $\begin{array}{c}0.12 \\
(0.21)\end{array}$ & $\begin{array}{c}0.03 \\
(0.05)\end{array}$ & $\begin{array}{c}0.06 \\
(0.11)\end{array}$ & $\begin{array}{c}0.65 \\
(1.41)\end{array}$ & $\begin{array}{c}0.66 \\
(1.76)\end{array}$ \\
\hline & $\mathrm{ik}$ & $\begin{array}{c}-11.12 \\
(-1.51) \\
{[0.04]}\end{array}$ & $\begin{array}{l}-21.78 \\
(-1.62) \\
{[0.13]}\end{array}$ & $\begin{array}{c}-37.23 \\
(-3.09) \\
{[0.25]}\end{array}$ & $\begin{array}{c}-47.68 \\
(-4.55) \\
{[0.36]}\end{array}$ & $\begin{array}{c}-45.72 \\
(-4.57) \\
{[0.38]}\end{array}$ & $\begin{array}{c}-48.38 \\
(-4.95) \\
{[0.46]}\end{array}$ \\
\hline
\end{tabular}

Nota: A tabela apresenta estatísticas de regressões in-sample multivariadas de previsão do prêmio de risco do mercado. As estão variáveis estão apresentadas na tabela 3.1. Para cada regressão a tabela reporta o corficiente de cada previsor, a estatística-t (entre parênteses)com a correção proposta em (29) e o R quadrado ajustado (entre colchetes). A amostra é trimestral do período 1981T1-2015T4. 


\section{2 \\ Previsão out-of-sample}

Nessa seção, eu utilizo a equação 3-1 para fazer previsão out-of-sample do prêmio de risco do mercado. Esse exercício é semelhante a previsão realtime (veja, por exemplo, (34)), pois utiliza apenas a informação disponível no momento da previsão. A diferença é que, caso haja correção posterior dos dados, as mesmas são incorporadas somente na previsão out-of-sample.

Para previsão out-of-sample eu utilizei as mesmas variáveis apresentadas na seção anterior. Além disso, conforme trabalhos anteriores (veja, (35)), propus duas versões para as variáveis que são desvio de um tendência. Para $\widehat{p t r}_{t}$ e $\widehat{c a y}_{t}$ o vetor de cointegração é recalculado a cada período. Para $\widehat{p t r} c_{t} \mathrm{e}$ $\widehat{\text { aay }} c_{t}$ o vetor de cointegração é calculados apenas uma vez. Como resultado, $\widehat{\operatorname{ptr} c} c_{t}$ e $\widehat{\text { cay }} c_{t}$ possuem um look-ahead bias e por isso é esperado tenham maior poder preditivo.

A tabela 3.4 mostra as seis estatísticas utilizadas para comparar a capacidade preditiva das variáveis. As três primeiras foram utilizadas em (17). A primeira é $R^{2}$ é calculada utilizando a equação 3-1 e toda a amostra disponível de cada variável. A segunda, $R_{I S}^{2}$ utiliza apenas os erros de 1990 a 2015. A terceira, $R_{O O S}^{2}$, compara o erro de previsão out-of-sample de 1990 a 2015 .

As três últimas estatísticas da tabela 3.4 foram propostas em (18). $R_{O O S, C O E F}^{2}$, corrige o coeficiente da regressão para zero sempre que o sinal do coeficiente é diferente do teórico. $R_{O O S, P R E M I O}^{2}$, corrige o prêmio previsto para zero sempre que a regressão prevê um prêmio negativo. $R_{O O S, C O E F, P R E M I O}^{2}$ faz a correção no coeficiente e se necessário faz a correção no prêmio.

As seis estatística da tabela 3.4 foram calculadas para previsão do prêmio de risco de $4,8,12,16,20$ e 24 trimestres. Apenas $i k_{t}$ foi melhor que $\widehat{p t r}_{t}$ em todos os períodos. Sobre as estatísticas de $\widehat{p t r}_{t}$, o $R^{2}$ aumentou com o aumento do horizonte de previsão. O $R_{I S}^{2}$ também aumentou com o horizonte de previsão. O $R_{O S S}^{2}$, conforme esperado, foi inferior a $R_{I S}^{2}$, mas também aumentou com o horizonte de previsão. Embora fosse esperado que $\widehat{p t r} c_{t}$ apresentasse estatísticas superiores a $\widehat{p t r}_{t}$, devido ao look-ahead bias e conforme aconteceu com $\widehat{c a y} c_{t}$ e $\widehat{c a y}_{t}$, não foi o que ocorreu. 


\begin{tabular}{|c|c|c|c|c|c|c|c|c|c|c|c|c|}
\hline \multicolumn{13}{|c|}{ Período de previsão $1991 \mathrm{~T} 1$ - 2015T4 } \\
\hline & ptr & ptrc & cay & cayc & $\mathrm{bm}$ & dfy & $\mathrm{d} / \mathrm{p}$ & ifl & $\mathrm{ik}$ & lty & tbl & tms \\
\hline \multicolumn{13}{|c|}{ Previsão de 4 trimestres } \\
\hline$R^{2}$ & $1,88 \%$ & - & 10,81 & - & 1,23 & 1,42 & 4,53 & 3,75 & 7,44 & 0,26 & 2,29 & 5,09 \\
\hline$R_{I S}^{2}$ & $4,53 \%$ & - & 13,61 & - & 0,42 & 1,41 & 2,80 & 5,07 & 10,41 & 0,23 & 1,88 & 2,99 \\
\hline$R_{O O S, P R E M I O}^{2}$ & $-1,41 \%$ & 0,07 & 9,24 & 16,11 & $-3,89$ & 0,57 & $-6,35$ & 4,73 & 9,52 & $-0,01$ & 1,57 & 2,10 \\
\hline$R_{O O S, C O E F, P R E M I O}^{2}$ & $0,53 \%$ & 2,02 & 9,24 & 16,11 & $-3,89$ & 0,57 & $-6,35$ & 4,73 & 9,52 & 0,52 & 1,57 & 2,10 \\
\hline \multicolumn{13}{|c|}{ Previsão de 8 trimestres } \\
\hline$R^{2}$ & 5,37 & - & 25,13 & - & 0,70 & 1,27 & 6,39 & 2,42 & 11,54 & 0,06 & 2,54 & 8,26 \\
\hline$R_{I S}^{2}$ & 8,88 & - & 33,89 & - & 0,26 & 2,65 & 5,32 & 3,79 & 20,71 & 0,19 & 6,27 & 15,22 \\
\hline$R_{O O S}^{2}$ & 4,60 & 5,23 & 18,65 & 30,99 & $-3,27$ & 1,22 & $-18,34$ & 3,18 & 17,83 & $-0,52$ & 4,27 & 13,23 \\
\hline$R^{2}$ & 7,94 & - & 34,85 & - & 0,24 & 1,33 & 6,80 & 1,91 & 20,64 & 0,00 & 3,23 & 13,52 \\
\hline$R_{I S}^{2}$ & 13,35 & - & 49,37 & - & 0,13 & 2,18 & 6,46 & 2,04 & 30,45 & $-0,10$ & 7,74 & 24,67 \\
\hline$R_{O O S}^{2}$ & 9,56 & 7,70 & 35,12 & 43,80 & $-2,37$ & 1,19 & $-12,78$ & 1,87 & 27,36 & $-0,63$ & 4,97 & 20,49 \\
\hline$R_{O O S, C O E F}^{2}$ & 4,42 & 9,67 & 35,12 & 43,80 & $-2,55$ & 0,43 & $-12,78$ & 1,87 & 27,36 & $-0,63$ & 4,97 & 20,49 \\
\hline$R_{O O S, P R E M I O}^{2}$ & 9,56 & 7,70 & 21,52 & 36,44 & $-2,37$ & 1,19 & $-13,06$ & 1,87 & 26,20 & $-0,63$ & 4,97 & 20,49 \\
\hline$R_{O O S, C O E F, P R E M I O}^{2}$ & 4,42 & 9,67 & 21,52 & 36,44 & $-2,55$ & 0,43 & $-13,06$ & 1,87 & 26,20 & $-0,63$ & 4,97 & 20,49 \\
\hline \multicolumn{13}{|c|}{ Previsão de 16 trismestres } \\
\hline$R^{2}$ & 11,06 & - & 36,24 & - & 0,31 & 2,55 & 7,59 & 2,68 & 31,33 & 0,08 & 3,07 & 16,44 \\
\hline$R_{I S}^{2}$ & 19,28 & - & 49,85 & - & 0,31 & 4,03 & 7,95 & 2,30 & 41,97 & $-0,07$ & 6,79 & 28,00 \\
\hline$R_{O O S}^{2}$ & 15,37 & 9,19 & 36,29 & 44,54 & $-2,15$ & 2,75 & $-10,06$ & 2,27 & 39,05 & $-0,46$ & 4,53 & 23,90 \\
\hline$R_{O O S, C O E F}^{2}$ & 8,68 & 12,70 & 36,29 & 44,54 & $-2,11$ & 2,74 & $-10,06$ & 2,27 & 39,05 & $-0,46$ & 4,53 & 23,90 \\
\hline$R_{O O S, P R E M I O}^{2}$ & 15,37 & 9,19 & 30,57 & 42,99 & $-2,15$ & 2,75 & $-11,10$ & 2,27 & 36,97 & $-0,46$ & 4,53 & 23,90 \\
\hline$R_{O O S, C O E F, P R E M I O}^{2}$ & 8,68 & 12,70 & 30,57 & 42,99 & $-2,11$ & 2,74 & $-11,10$ & 2,27 & 36,97 & $-0,46$ & 4,53 & 23,90 \\
\hline \multicolumn{13}{|c|}{ Previsão de 20 trimestres } \\
\hline$R_{I S}^{2}$ & 28,76 & - & 25,06 & - & 0,78 & 10,10 & 14,10 & 1,24 & 48,60 & 1,49 & 1,51 & 24,02 \\
\hline$R_{O O S}^{2}$ & 25,33 & 21,79 & 4,70 & 23,73 & $-4,42$ & 8,41 & $-24,64$ & 0,43 & 45,41 & 0,68 & $-0,81$ & 21,01 \\
\hline$R_{O O S, C O E F}^{2}$ & 25,35 & 21,84 & 4,70 & 23,73 & $-4,51$ & 8,41 & $-24,64$ & 0,43 & 45,41 & 0,68 & $-3,12$ & 21,01 \\
\hline$R_{O O S, P R E M I O}^{2}$ & 25,33 & 21,79 & 16,78 & 27,95 & $-4,42$ & 8,41 & $-24,58$ & 0,43 & 45,13 & 0,68 & $-0,81$ & 21,01 \\
\hline$R_{O O S, C O E F, P R E M I O}^{2}$ & 25,35 & 21,84 & 16,78 & 27,95 & $-4,51$ & 8,41 & $-24,58$ & 0,43 & 45,13 & 0,68 & $-3,12$ & 21,01 \\
\hline \multicolumn{13}{|c|}{$\begin{array}{l}\text { Nota: A tabela apresenta estatísticas de previsão da regressão univariada do prêmio de risco do mercado em diferentes previsores. O prêmio de risco de mercado é o } \\
\text { excesso de retorno do índice CRSP value-weight em relação ao 3-month treasury bill. As variáveis utilizadas como previsores são as mesmas apresentadas na tabela } \\
\text { 3.1. } R^{2} \text { é a estatística da regressão OLS calculada utilizando toda a amostra disponível; } R_{I S}^{2} \text { é a estatística da regressão OLS calculada utilizando toda amostra, mas } \\
\text { que só considera os erros do período de } 1991 T 1-2015 T 4 ; R_{O S}^{2} \text { e as próximas estatísticas comparam o erro de previsão da regressão OLS recalculada a cada trimestre } \\
\text { e, além disso, consideram apenas os erros do período de } 1991 \mathrm{~T} 1-2015 \mathrm{~T} 4 ; R_{O O S, C O E F}^{2} \text { é calculada considerando o coeficiente angular da regressão OLS como zero } \\
\text { quando seu sinal (positivo ou negativo) é diferente do teórico. } R_{O O S, P R E M I O}^{2} \text { considera o premio como zero quando seu sinal é negativo; } R_{O O S, C O E F, P R E M I O}^{2} \text { é } \\
\text { calculada primeiro fazendo a correção do coeficiente angular da regressão OLS e em seguida, se necessário, é feita a correção do prêmio de risco. }\end{array}$} \\
\hline
\end{tabular}

As correção do prêmio de risco e do coeficiente propostas em (18) aumentaram as estatísticas de $\widehat{p t r} c_{t}$ em todas os horizontes de previsão, mas não as de $\widehat{p t r}_{t}$. Por exemplo, para a previsão de 20 trimestres o $R_{O S S}^{2}$ de $\widehat{p t r} c_{t}$ era $19,76 \%$. Com a correção, o $R_{O S S, C O E F, P R E M I O}^{2}$ foi para 20,52\%. Já para $\widehat{p t r}_{t}$ o $R_{O S S}^{2}$ era $24,10 \%$, com a correção, o $R_{O S S}^{2}$ foi de $21,32 \%$.

A tabela 3.5 apresenta o resultado do teste MDM de comparação direta entre as variáveis, proposto em (36). Esse teste é construído avaliando se a diferença do erro de previsão de $\widehat{p t r}$ (ou $\widehat{p t c}$ ) e da variável competidora está correlacionado com o erro de previsão da variável competidora ${ }^{5}$. Dessa forma, a hipótese H0 supõe que toda informação de $\widehat{p t r}$ (ou $\widehat{p t r}$ ) relevante para previsão está contida na variável competidora e, portanto, $\widehat{p t r}$ (ou $\widehat{p t r c}$ ) é englobada pela

${ }^{5}$ No cáculo da estística Diebold-Marino foi feita a correção para autocorrelação devida a sobreposição dos períodos, utilizando o método de Newey-West 
variável competidora. H1 supõe que $\widehat{p t r}$ (ou $\widehat{p t r c}$ ) não é englobada.

No painel A da tabela 3.5 são apresentados os resultados do teste que avaliou se $\widehat{p t r}$ é englobada e no painel B são apresentados os resultados do teste que avaliou se $\widehat{\operatorname{tr} c}$ é englobada. No painel A, na primeira linha da previsão de cada período é mostrada a razão da raiz da soma do quadrado dos erros de previsão de $\widehat{p t r}$ sobre a raiz da soma do quadrado dos erros de previsão de outros previsores. A segunda linha mostra a estatística modified DieboldMariano. A terceira linha mostra o p-valor do modified Diebold-Mariano. Em negrito estão os casos nos quais H0 é rejeitada a 10\%. A tabela mostra que na previsão do prêmio de risco de 5 e 6 anos, a um nível de significância de 10\%, $\widehat{p t r}$ e $\widehat{p t r} c$ não são englobadas por $\widehat{c a y}, \mathrm{bm}, \mathrm{dfy}, \mathrm{d} / \mathrm{p}$, ifl, lty e tbl.

Na tabela 3.6 eu avalio a capacidade de $\widehat{p t r}$ prever e ser previsto pelas variáveis competidoras no horizonte de 1 a 4 trimestres. No painel A são apresentadas as estatísticas t e $R^{2}$ das regressões quando $\widehat{p t r}$ é utilizado como previsor. A estatística-t do coeficiente de $\widehat{p t r}$ foi maior em todos os períodos na previsão de ik e tms, variáveis ique apresentaram bons resultados de previsão in-sample e out-of-sample. No painel B, quando $\widehat{p t r}$ é previsto, a estatística-t foi maior em todos os períodos quando ik e tms foram utilizados como previsores. 
Tabela 3.5: Comparação entre $\widehat{p t r}$ e outros previsores

\begin{tabular}{|c|c|c|c|c|c|c|c|c|c|c|c|}
\hline \multicolumn{12}{|c|}{ Painel A: regressão de cointegração restimada a cada trimestre } \\
\hline$\widehat{p t r}$ & $\widehat{p t r c}$ & $\widehat{c a y}_{t}$ & $\widehat{c a y} c_{t}$ & $\mathrm{bm}$ & dfy & $\mathrm{d} / \mathrm{p}$ & ifl & $\mathrm{ik}$ & lty & tbl & tms \\
\hline \multicolumn{12}{|c|}{ Previsão de 1 ano } \\
\hline- & 1,01 & 1,02 & 1,16 & 0,98 & 1,02 & 0,83 & 1,06 & 1,12 & 1,01 & 1,03 & 1,04 \\
\hline- & $(-0,29)$ & $(-1,65)$ & $(-0,47)$ & $(-1,40)$ & $(-0,68)$ & $(-2,44)$ & $(0,40)$ & $(0,04)$ & $(-0,56)$ & $(-0,13)$ & $(-0,39)$ \\
\hline- & $78 \%$ & $10 \%$ & $64 \%$ & $17 \%$ & $50 \%$ & $2 \%$ & $69 \%$ & $97 \%$ & $57 \%$ & $90 \%$ & $70 \%$ \\
\hline \multicolumn{12}{|c|}{ Previsão de 2 anos } \\
\hline- & 1,01 & 1,17 & 1,38 & 0,92 & 0,97 & 0,81 & 0,99 & 1,16 & 0,95 & 1,00 & 1,10 \\
\hline- & $(-0,02)$ & $(-1,51)$ & $(-0,15)$ & $(-1,48)$ & $(-1,01)$ & $(-2,17)$ & $(-0,76)$ & $(0,12)$ & $(-1,17)$ & $(-0,59)$ & $(0,16)$ \\
\hline- & $98 \%$ & $13 \%$ & $88 \%$ & $14 \%$ & $32 \%$ & $3 \%$ & $45 \%$ & $91 \%$ & $25 \%$ & $55 \%$ & $88 \%$ \\
\hline \multicolumn{12}{|c|}{ Previsão de 3 anos } \\
\hline- & 0,98 & 1,39 & 1,61 & 0,88 & 0,92 & 0,80 & 0,92 & 1,25 & 0,90 & 0,95 & 1,14 \\
\hline- & $(-1,04)$ & $(-1,77)$ & $(0,14)$ & $(-1,69)$ & $(-1,34)$ & $(-2,03)$ & $(-1,31)$ & $(-0,23)$ & $(-1,49)$ & $(-1,04)$ & $(-0,06)$ \\
\hline- & $30 \%$ & $8 \%$ & $89 \%$ & $9 \%$ & $18 \%$ & $5 \%$ & $19 \%$ & $82 \%$ & $14 \%$ & $30 \%$ & $95 \%$ \\
\hline \multicolumn{12}{|c|}{ Previsão de 4 anos } \\
\hline- & 0,93 & 1,33 & 1,53 & 0,83 & 0,87 & 0,77 & 0,87 & 1,39 & 0,84 & 0,89 & 1,11 \\
\hline- & $(-1,51)$ & $(-2,25)$ & $(-0,07)$ & $(-1,77)$ & $(-1,37)$ & $(-2,01)$ & $(-1,50)$ & $(-0,42)$ & $(-1,58)$ & $(-1,28)$ & $(-0,23)$ \\
\hline- & $14 \%$ & $3 \%$ & $95 \%$ & $8 \%$ & $17 \%$ & $5 \%$ & $14 \%$ & $68 \%$ & $12 \%$ & $20 \%$ & $82 \%$ \\
\hline \multicolumn{12}{|c|}{ Previsão de 5 anos } \\
\hline- & 0,95 & 1,05 & 1,24 & 0,73 & 0,82 & 0,67 & 0,78 & 1,33 & 0,76 & 0,78 & 0,99 \\
\hline- & $(-1,32)$ & $(-2,83)$ & $(-0,63)$ & $(-2,13)$ & $(-1,43)$ & $(-2,30)$ & $(-2,15)$ & $(-0,74)$ & $(-1,96)$ & $(-1,95)$ & $(-0,56)$ \\
\hline - & $19 \%$ & $1 \%$ & $53 \%$ & $4 \%$ & $16 \%$ & $2 \%$ & $3 \%$ & $46 \%$ & $5 \%$ & $5 \%$ & $57 \%$ \\
\hline \multicolumn{12}{|c|}{ Previsão de 6 anos } \\
\hline- & 0,95 & 0,78 & 0,98 & 0,72 & 0,82 & 0,60 & 0,75 & 1,37 & 0,75 & 0,74 & 0,95 \\
\hline- & $(-0,85)$ & $(-3,45)$ & $(-1,48)$ & $(-1,78)$ & $(-1,21)$ & $(-2,29)$ & $(-1,93)$ & $(-0,96)$ & $(-1,76)$ & $(-1,84)$ & $(-0,88)$ \\
\hline- & $40 \%$ & $0 \%$ & $14 \%$ & $8 \%$ & $23 \%$ & $2 \%$ & $6 \%$ & $34 \%$ & $8 \%$ & $7 \%$ & $38 \%$ \\
\hline \multicolumn{12}{|c|}{ Painel B: regressão de cointegração fixa } \\
\hline$\widehat{p t r}$ & $\widehat{p t r c}$ & $\widehat{\operatorname{cay}}_{t}$ & $\widehat{\operatorname{cay}} c_{t}$ & $\mathrm{bm}$ & dfy & $\mathrm{d} / \mathrm{p}$ & ifl & $\mathrm{ik}$ & lty & tbl & tms \\
\hline \multicolumn{12}{|c|}{ Previsão de 1 ano } \\
\hline 0,99 & - & 1,01 & 1,14 & 0,96 & 1,01 & 0,82 & 1,05 & 1,11 & 1,00 & 1,02 & 1,02 \\
\hline$(-0,85)$ & - & $(-1,57)$ & $(-0,53)$ & $(-1,19)$ & $(-0,47)$ & $(-2,32)$ & $(0,67)$ & $(0,05)$ & $(-0,43)$ & $(-0,08)$ & $(-0,67)$ \\
\hline $40 \%$ & - & $12 \%$ & $60 \%$ & $24 \%$ & $64 \%$ & $2 \%$ & $51 \%$ & $96 \%$ & $67 \%$ & $94 \%$ & $51 \%$ \\
\hline \multicolumn{12}{|c|}{ Previsão de 2 anos } \\
\hline 0,99 & - & 1,16 & 1,37 & 0,92 & 0,96 & 0,80 & 0,98 & 1,15 & 0,94 & 0,99 & 1,09 \\
\hline$(-0,42)$ & - & $(-1,44)$ & $(-0,08)$ & $(-1,55)$ & $(-1,04)$ & $(-2,21)$ & $(-0,79)$ & $(0,19)$ & $(-1,22)$ & $(-0,59)$ & $(0,23)$ \\
\hline $68 \%$ & - & $15 \%$ & $94 \%$ & $12 \%$ & $30 \%$ & $3 \%$ & $43 \%$ & $85 \%$ & $22 \%$ & $56 \%$ & $82 \%$ \\
\hline \multicolumn{12}{|c|}{ Previsão de 3 anos } \\
\hline 1,02 & - & 1,42 & 1,64 & 0,90 & 0,93 & 0,82 & 0,94 & 1,27 & 0,92 & 0,97 & 1,16 \\
\hline$(-0,40)$ & - & $(-1,33)$ & $(0,53)$ & $(-1,56)$ & $(-1,14)$ & $(-1,91)$ & $(-1,10)$ & $(0,09)$ & $(-1,30)$ & $(-0,75)$ & $(0,48)$ \\
\hline $69 \%$ & - & $19 \%$ & $60 \%$ & $12 \%$ & $26 \%$ & $6 \%$ & $27 \%$ & $92 \%$ & $20 \%$ & $46 \%$ & $63 \%$ \\
\hline \multicolumn{12}{|c|}{ Previsão de 4 anos } \\
\hline 1,07 & - & 1,43 & 1,64 & 0,89 & 0,93 & 0,83 & 0,93 & 1,4 & 0,90 & 0,95 & 1,19 \\
\hline$(0,38)$ & - & $(-1,69)$ & $(0,69)$ & $(-1,51)$ & $(-1,06)$ & $(-1,84)$ & $(-1$, & $(0$, & $(-1$ & $(-0$ & $(0,53)$ \\
\hline $70 \%$ & - & $9 \%$ & $49 \%$ & $14 \%$ & $29 \%$ & $7 \%$ & $24 \%$ & $99 \%$ & $21 \%$ & $37 \%$ & $60 \%$ \\
\hline \multicolumn{12}{|c|}{ Previsão de 5 anos } \\
\hline 1,06 & - & 1,11 & 1,31 & 0,78 & 0,87 & 0,70 & 0,82 & 1,40 & 0,80 & 0,82 & 1,04 \\
\hline$(0,55)$ & - & $(-2,49)$ & $(-0,16)$ & $(-2,03)$ & $(-1,37)$ & $(-2,21)$ & $(-2,09)$ & $(-0,50)$ & $(-1,88)$ & $(-1,91)$ & $(-0,22)$ \\
\hline $58 \%$ & - & $1 \%$ & $87 \%$ & $5 \%$ & $17 \%$ & $3 \%$ & $4 \%$ & $62 \%$ & $6 \%$ & $6 \%$ & $83 \%$ \\
\hline \multicolumn{12}{|c|}{ Previsão de 6 anos } \\
\hline 1,05 & - & 0,82 & 1,03 & 0,75 & 0,85 & 0,63 & 0,79 & 1,43 & 0,79 & 0,78 & 0,99 \\
\hline$(-0,01)$ & - & $(-2,75)$ & $(-1,19)$ & $(-1,48)$ & $(-1,10)$ & $(-2,07)$ & $(-1,51)$ & $(-0,80)$ & $(-1,51)$ & $(-1,48)$ & $(-0,66)$ \\
\hline $100 \%$ & - & $1 \%$ & $24 \%$ & $14 \%$ & $28 \%$ & $4 \%$ & $14 \%$ & $43 \%$ & $14 \%$ & $14 \%$ & $51 \%$ \\
\hline
\end{tabular}

Nota: A tabela reporta se outros previsores propostos na literatura englobam $\widehat{p t r}$. O painel A informa a razão da raiz da soma do quadrado dos erros de previsão de $\widehat{p t r}$ sobre a raiz da soma do quadrado dos erros de previsão de outros previsores; Entre parenteses está a estatística modified Diebold-Mariano proposta por (36); o p-valor do modified Diebold-Mariano é apresentado em seguida, onde H0 suponhe que $\widehat{p t r}$ é englobada e H1 suponhe que $\widehat{p t r}$ não é englobada. 
Tabela 3.6: Relação de previsão entre $\widehat{p t r}_{t}$ e as outras variáveis

\begin{tabular}{|c|c|c|c|c|c|c|c|c|c|}
\hline \multicolumn{10}{|c|}{ Painel A: $\widehat{p t r}_{t}$ como previsor } \\
\hline & cay & $\mathrm{ik}$ & $\mathrm{bm}$ & tbl & $\ln (\mathrm{d} / \mathrm{p})$ & lty & tms & ifl & dfy \\
\hline \multicolumn{10}{|c|}{ Previsão 1 trimestre } \\
\hline estatística-t & $-0,95$ & $-7,91$ & 0,52 & $-3,16$ & 1,89 & $-1,16$ & 5,91 & $-0,47$ & $-0,95$ \\
\hline$R^{2}$ & $0,65 \%$ & $31,36 \%$ & $0,20 \%$ & $6,78 \%$ & $2,53 \%$ & $0,98 \%$ & $20,31 \%$ & $0,16 \%$ & $0,66 \%$ \\
\hline \multicolumn{10}{|c|}{ Previsão de 2 trimestres } \\
\hline estatística-t & $-0,93$ & $-7,59$ & 0,63 & $-3,23$ & 1,82 & $-1,02$ & 6,55 & 0,01 & $-1,64$ \\
\hline$R^{2}$ & $0,64 \%$ & $29,75 \%$ & $0,29 \%$ & $7,11 \%$ & $2,38 \%$ & $0,76 \%$ & $24,00 \%$ & $0,00 \%$ & $1,93 \%$ \\
\hline \multicolumn{10}{|c|}{ Previsão de 3 trimestres } \\
\hline estatística-t & $-0,95$ & $-7,02$ & 0,68 & $-3,35$ & 1,76 & $-1,04$ & 6,65 & $-0,25$ & $-1,71$ \\
\hline$R^{2}$ & $0,67 \%$ & $26,75 \%$ & $0,34 \%$ & $7,68 \%$ & $2,24 \%$ & $0,79 \%$ & $24,66 \%$ & $0,05 \%$ & $2,13 \%$ \\
\hline \multicolumn{10}{|c|}{ Previsão de 4 trimestres } \\
\hline estatística-t & $-0,85$ & $-6,41$ & 0,80 & $-2,94$ & 1,82 & $-0,93$ & 5,52 & 0,00 & $-2,03$ \\
\hline$R^{2}$ & $0,54 \%$ & $23,46 \%$ & $0,48 \%$ & $6,05 \%$ & $2,41 \%$ & $0,64 \%$ & $18,53 \%$ & $0,00 \%$ & $2,99 \%$ \\
\hline \multicolumn{10}{|c|}{ Painel B: $\widehat{p t r}_{t}$ sendo previsto } \\
\hline & cay & $\mathrm{ik}$ & $\mathrm{bm}$ & tbl & $\ln (\mathrm{d} / \mathrm{p})$ & lty & tms & ifl & dfy \\
\hline \multicolumn{10}{|c|}{ Previsão 1 trimestre } \\
\hline estatística-t & $-0,38$ & $-7,62$ & 0,17 & $-3,25$ & 1,90 & $-1,51$ & 5,20 & $-1,04$ & $-0,03$ \\
\hline$R^{2}$ & $0,11 \%$ & $29,78 \%$ & $0,02 \%$ & $7,15 \%$ & $2,57 \%$ & $1,63 \%$ & $16,49 \%$ & $0,79 \%$ & $0,00 \%$ \\
\hline \multicolumn{10}{|c|}{ Previsão de 2 trimestres } \\
\hline estatística-t & 0,11 & $-6,87$ & 0,12 & $-2,94$ & 2,01 & $-1,47$ & 4,36 & $-1,59$ & 0,23 \\
\hline$R^{2}$ & $0,01 \%$ & $25,78 \%$ & $0,01 \%$ & $5,96 \%$ & $2,89 \%$ & $1,56 \%$ & $12,28 \%$ & $1,82 \%$ & $0,04 \%$ \\
\hline \multicolumn{10}{|c|}{ Previsão de 3 trimestres } \\
\hline estatística-t & 0,49 & $-5,84$ & 0,01 & $-2,44$ & 1,98 & $-1,26$ & 3,49 & $-1,19$ & 0,25 \\
\hline$R^{2}$ & $0,17 \%$ & $20,15 \%$ & $0,00 \%$ & $4,22 \%$ & $2,82 \%$ & $1,16 \%$ & $8,27 \%$ & $1,04 \%$ & $0,05 \%$ \\
\hline \multicolumn{10}{|c|}{ Previsão de 4 trimestres } \\
\hline estatística-t & 0,96 & $-4,84$ & 0,06 & $-1,90$ & 2,08 & $-0,98$ & 2,70 & $-0,74$ & 0,40 \\
\hline & $0,68 \%$ & $14,86 \%$ & $0,00 \%$ & $2,62 \%$ & $3,12 \%$ & $0,71 \%$ & $5,17 \%$ & $0,40 \%$ & $0,12 \%$ \\
\hline
\end{tabular}

Nota: No painel A são apresentadas as estatísticas t e $R^{2}$ das regressões quando $\widehat{p t r}$ é utilizado como previsor. No painel B são apresentadas as estatísticas t e $R^{2}$ quando $\widehat{p t r}$ é previsto. 
A figura 3.1, inspirada em (17), mostra a performance in-sample e out-ofsample da variável $\widehat{p t r}_{t}$ e a performance out-of-sample de $\widehat{p t r}_{t}$ na previsão do prêmio de risco do mercado. Para a regressão out-of-sample, o gráfico mostra a soma acumulada dos erros quadráticos utilizando a média corrente do prêmio de risco como previsor, menos a soma acumulada dos erros quadráticos de previsão utilizando dados correntes $\widehat{p t r}_{t}$. Para a regressão in-sample, o gráfico mostra a soma acumulada do quadrado do prêmio de risco subtraído da sua média de todo o período, menos a soma acumulada dos erros quadráticos de previsão utilizando toda a amostra do $\widehat{p t r}_{t}$. No gráfico, sempre que o traçado está subindo significa que é melhor usar $\widehat{p t r}_{t}$ em relação a média do prêmio de risco. Sempre que o traçado está descendo é porque é melhor usar média do prêmio de risco como previsor.

Pelo gráfico, $\widehat{p t r}_{t}$ apresentou capacidade preditiva superior a média do prêmio de risco nos períodos 1990-1995 e após 2008. $\widehat{p t r}_{t}$ foi superior a $\widehat{p t r}_{t}$, na previsão out-of-sample, na maior parte do período, o que era esperado devido ao look-ahead bias de $\widehat{p t r} c_{t}$. Entretanto, no último ano, $\widehat{p t r}_{t}$ foi melhor que $\widehat{\operatorname{ptr}} c_{t}$. Essa situação foi inesperada e pode ser devido a crise de 2008, quando houve uma forte mudança dos dados.

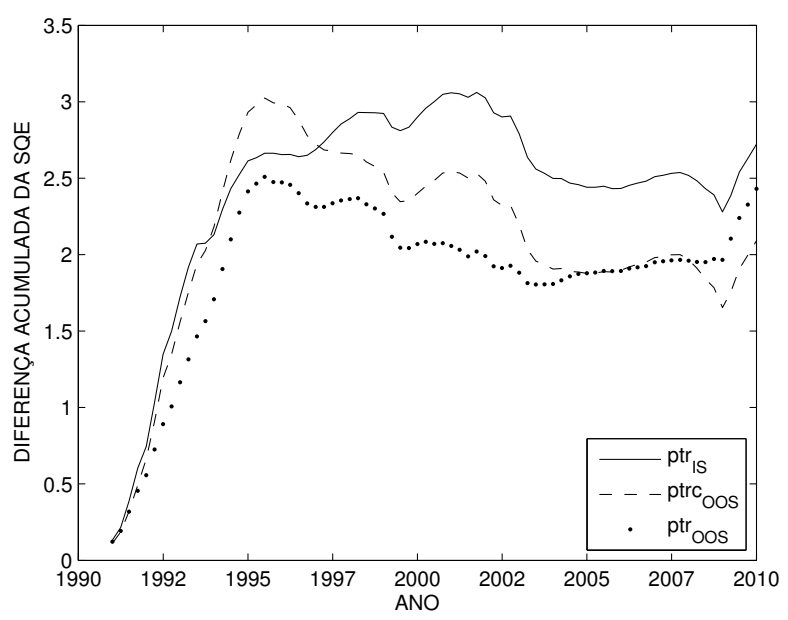

Figura 3.1: Performance de $\widehat{p t r}_{t}$ e $\widehat{p t r} c_{t}$ na previsão do retorno de seis anos do prêmio de risco do mercado 


\section{4 \\ Conclusão}

Nesse estudo eu calculo uma proxy para a propensão à tomada de risco dos jogadores de cassino a partir do desvio da tendência de longo prazo que existe entre gastos com apostas em cassinos, produto interno bruto, custo médio de viajar e aumento da oferta de cassinos. Em seguida, eu utilizo essa proxy para prever o prêmio de risco do mercado em regressões in-sample e out-of-sample

Conforme esperado, os resultados da previsão out-of-sample foram inferiores aos resultados da previsão in-sample. E, além disso, as estatísticas de previsão aumentaram com o horizonte de previsão. Os resultados da previsão in-sample, por exemplo, apresentaram estatística t significante apenas em horizontes longos de previsão: 16, 20 e 24 semestres.

A propensão à tomada de risco apresentou bons resultados na previsão do prêmio de risco do mercado e até pode ser utilizada em conjunto com outros previsores sugeridos na literatura para que sejam obtidos melhores estatísticas de previsão. Entretanto, o Investment to Capital Ratio conseguiu superá-lo em todos os horizontes. 


\section{Referências bibliográficas}

1 YUEN, K. S.; LEE, T. M. Could mood state affect risk-taking decisions? Journal of affective disorders, Elsevier, v. 75, n. 1, p. 11-18, 2003.

2 ISEN, A. M.; PATRICK, R. The effect of positive feelings on risk taking: When the chips are down. Organizational behavior and human performance, Elsevier, v. 31, n. 2, p. 194-202, 1983.

3 CHEN, Y.; KUMAR, A.; ZHANG, C. Searching for gambles: Investor attention, gambling sentiment, and stock market outcomes. 2016.

4 MIKESELL, J. L. State lottery sales and economic activity. National Tax Journal, JSTOR, p. 165-171, 1994.

5 KUMAR, A. Who gambles in the stock market? The Journal of Finance, Wiley Online Library, v. 64, n. 4, p. 1889-1933, 2009.

6 LEVITZKY, I.; ASSANE, D.; ROBINSON, W. Determinants of gaming revenue: extent of changing attitudes in the gaming industry. Applied economics letters, Taylor \& Francis, v. 7, n. 3, p. 155-158, 2000.

7 PHILANDER, K. S. The effect of online gaming on commercial casino revenue. UNLV Gaming Research \& Review Journal, Board of Regents of the University and the Community College System of Nevada on behalf of the University of Nevada, Las Vegas, v. 15, n. 2, p. 23, 2011.

8 HORVÁTH, C.; PAAP, R. The effect of recessions on gambling expenditures. Journal of Gambling Studies, Springer, v. 28, n. 4, p. 703-717, 2012.

9 BARBERIS, N. A model of casino gambling. Management Science, INFORMS, v. 58, n. 1, p. 35-51, 2012.

10 TVERSKY, A.; KAHNEMAN, D. Advances in prospect theory: Cumulative representation of uncertainty. Journal of Risk and uncertainty, Springer, v. 5, n. 4, p. 297-323, 1992.

11 SUHONEN, N.; SAASTAMOINEN, J.; LINDEN, M. A dual theory approach to estimating risk preferences in the parimutuel betting market. Empirical Economics, Springer, p. 1-17, 2017.

12 CHETTY, R. A new method of estimating risk aversion. The American Economic Review, JSTOR, v. 96, n. 5, p. 1821-1834, 2006.

13 GUISO, L.; SAPIENZA, P.; ZINGALES, L. Time varying risk aversion. [S.I.], 2013. 
14 LETTAU, M.; LUDVIGSON, S. Consumption, aggregate wealth, and expected stock returns. the Journal of Finance, Wiley Online Library, v. 56, n. 3, p. 815-849, 2001.

15 JOHANSEN, S. Statistical analysis of cointegration vectors. Journal of economic dynamics and control, Elsevier, v. 12, n. 2-3, p. 231-254, 1988.

16 STOCK, J. H.; WATSON, M. W. A simple estimator of cointegrating vectors in higher order integrated systems. Econometrica: Journal of the Econometric Society, JSTOR, p. 783-820, 1993.

17 WELCH, I.; GOYAL, A. A comprehensive look at the empirical performance of equity premium prediction. Review of Financial Studies, Soc Financial Studies, v. 21, n. 4 , p. $1455-1508,2008$.

18 CAMPBELL, J. Y.; THOMPSON, S. B. Predicting excess stock returns out of sample: Can anything beat the historical average? The Review of Financial Studies, Society for Financial Studies, v. 21, n. 4, p. 1509-1531, 2007.

19 COCHRANE, J. H. Production-based asset pricing and the link between stock returns and economic fluctuations. The Journal of Finance, Wiley Online Library, v. 46, n. 1, p. 209-237, 1991.

20 KOTHARI, S. P.; SHANKEN, J. Book-to-market, dividend yield, and expected market returns: A time-series analysis. Journal of Financial Economics, Elsevier, v. 44, n. 2, p. 169-203, 1997.

21 PONTIFF, J.; SCHALL, L. D. Book-to-market ratios as predictors of market returns. Journal of Financial Economics, Elsevier, v. 49, n. 2, p. 141-160, 1998.

22 HODRICK, R. J. Dividend yields and expected stock returns: Alternative procedures for inference and measurement. Review of Financial studies, Soc Financial Studies, v. 5, n. 3, p. 357-386, 1992.

23 CAMPBELL, J. Y. Stock returns and the term structure. Journal of financial economics, Elsevier, v. 18, n. 2, p. 373-399, 1987.

24 FAMA, E. F.; FRENCH, K. R. Business conditions and expected returns on stocks and bonds. Journal of financial economics, Elsevier, v. 25, n. 1, p. 23-49, 1989.

25 KEIM, D. B.; STAMBAUGH, R. F. Predicting returns in the stock and bond markets. Journal of financial Economics, Elsevier, v. 17, n. 2, p. 357-390, 1986.

26 CAMPBELL, J. Y.; VUOLTEENAHO, T. Inflation illusion and stock prices. [S.I.], 2004.

27 FAMA, E. F. Stock returns, real activity, inflation, and money. The American Economic Review, JSTOR, v. 71, n. 4, p. 545-565, 1981.

28 LINTNER, J. Inflation and security returns. The Journal of Finance, Wiley Online Library, v. 30, n. 2, p. 259-280, 1975. 
29 NEWEY, W. K.; WEST, K. D. Automatic lag selection in covariance matrix estimation. The Review of Economic Studies, Oxford University Press, v. 61, n. 4, p. 631-653, 1994.

30 FAMA, E. F.; FRENCH, K. R. Dividend yields and expected stock returns. Journal of financial economics, Elsevier, v. 22, n. 1, p. 3-25, 1988.

31 COCHRANE, J. H. Asset Pricing:(Revised Edition). [S.I.]: Princeton university press, 2009.

32 BOUDOUKH, J.; RICHARDSON, M.; WHITELAW, R. F. The myth of long-horizon predictability. The Review of Financial Studies, Society for Financial Studies, v. 21, n. 4, p. 1577-1605, 2006.

33 FERSON, W. E.; SARKISSIAN, S.; SIMIN, T. T. Spurious regressions in financial economics? The Journal of Finance, Wiley Online Library, v. 58, n. 4, p. 1393-1413, 2003.

34 GARCIA, M. G.; MEDEIROS, M. C.; VASCONCELOS, G. F. Real-time inflation forecasting with high-dimensional models: The case of brazil. International Journal of Forecasting, Elsevier, v. 33, n. 3, p. 679-693, 2017.

35 BRENNAN, M. J.; XIA, Y. Tay's as good as cay. Finance Research Letters, Elsevier, v. 2, n. 1, p. 1-14, 2005.

36 HARVEY, D. S.; LEYBOURNE, S. J.; NEWBOLD, P. Tests for forecast encompassing. Journal of Business \& Economic Statistics, Taylor \& Francis Group, v. 16, n. 2, p. 254-259, 1998.

37 THOMPSON, W. N. Gambling in America: An Encyclopedia of History, Issues, and Society. [S.I.]: ABC-CLIO, 2015. 
A

\section{Janela temporal da estimação de ptr}

O uso de dados a partir 1981 para a estimação de $\widehat{p t r}_{t}$ se deve a vários motivos, por exemplo: em 1978 foi publicada a lei de desregulamentação das companhias aéreas dos Estados Unidos. Essa lei tinha como objetivo a introdução do livre mercado aéreo em quatro anos. A partir dessa lei, ocorreu um grande aumento do número de voos e no número de companhias aéreas, fazendo com que o transporte aéreo americano passasse a operar em outra patamar. Um segundo motivo é que em novembro de 1980 um incêndio ${ }^{1}$ destruiu o MGM Grand ${ }^{2}$ cassino-hotel em Las Vegas, isso aconteceu dois anos após abrir o primeiro cassino fora do estado Nevada. Um terceiro motivo é que pelo teste de quebra estrutural de Quandt-Andrews, apresentado na tabela A.1, há quebra estrutural a significância de $1 \%$ e o período mais provável de quebra estrutural é 1981Q2. Um quarto motivo é que a variância da variação percentual da variável $\mathrm{gac}_{t}$ do período de 1959T1 até 1980T4 é 2,5 vezes maior que a variação percentual o período de $1981 \mathrm{~T} 1$ até $2016 \mathrm{~T} 4$.

Tabela A.1: Teste de quebra estrutural de QuandtAndrews

\begin{tabular}{lcc}
\hline Estatística & Valor & p-valor \\
\hline Maximum LR F-statistic (1981Q2) & 102,65 & 0,00 \\
Maximum Wald F-statistic (1981Q2) & 410,59 & 0,00 \\
Exp LR F-statistic & 47,89 & 0,00 \\
Exp Wald F-statistic & 201,07 & 0,00 \\
Ave LR F-statistic & 57,36 & 0,00 \\
Ave Wald F-statistic & 229,44 & 0,00 \\
\hline
\end{tabular}

Nota: O teste foi realizado utilizando dados trimestrais de 1959 T1 até 2016T4 e retirando os primeiros e últimos 30\% da amostra, todas as estatísticas de teste rejeitaram a hipótese nula de que não há quebra estrutural ao nível de significância de 1\%. Além disso, o teste de Quant-Andrews indicou 1981Q2 como a data mais provável de quebra estrutural na regressão gac $c_{t}=\alpha+\beta_{P I B} p i b_{t}+\beta_{C V} c v_{t}+$ $\beta_{L C} L C_{t}$.

\footnotetext{
${ }^{1}$ Esse foi o incêndio em hotel que teve o segundo maior número de mortes na história dos Estados Unidos. fonte: http:fire.co.clark.nv.us(S(noukm0w4eis35iej5apbv3um))/MGM.aspx

${ }^{2}$ Quando construído em 1973 esse era o maior(37) hotel do mundo
} 
B

\section{Detalhes das variáveis}

O Gasto com Aposta em Cassinos ( $\left.g a c_{t}\right)$ é o logaritmo do total gasto com apostas nos cassinos dos Estados Unidos. Essa variável trimestral é calculada a partir da série casino gambling, fornecida pelo BEA (Bureau of Economic Analisys), deflacionada pelo seu índice de preço e divida pela população dos Estados Unidos. Para construção da série casino gambling o BEA coleta dados de quatorze estados a partir das Agências de Controle de Jogos (State Gaming Control Comissions) dos estados e do Census $Q S S^{1}$.

O Produto Interno Bruto $\left(p i b_{t}\right)$ é o controle para a variação do gasto com apostas devido a mudanças na renda populacional. Essa variável é calculada a partir do logaritmo da série Gross Domestic Product, fornecida pelo BEA, deflacionada pelo seu índice de preço e dividida pela população dos Estados Unidos ${ }^{2}$.

O Custo de Viajar $\left(c v_{t}\right)$ é a variável controle para as mudanças no custo de viajar nos Estados Unidos. Essa variável é o logarítmo do índice de preço air transportation ${ }^{3}$, fornecido pelo BEA, dividido pelo índice de inflação dos Estados Unidos, fornecido pelo BLS Consumer Price Index ${ }^{4}$.

O Número de Estados que Legalizaram Cassinos $\left(L C_{t}\right)$ é o controle para evolução da oferta de cassinos nos Estados Unidos. Essa variável conta o número de estados que legalizaram o cassino em um determinado trimestre, considerando apenas os quatorze estados que o BEA utiliza para o computo da séria casino gambling.

Nos Estados Unidos, cabe aos estados legalizar e regular as operações de cassinos. Desde 1931, os estados têm legalizado os cassinos e legislado sobre o assunto. Como cada estado possui legislação própria, cada legislação tem a

\footnotetext{
1 fonte: casino gambling - BEA tabela 2.4.5U, linha 221. índice de preço casino gambling - BEA tabela 2.4.4U, linha 221. população dos Estados Unidos - BEA tabela 2.1, linha 40.

${ }^{2}$ Fonte: Gross Domestic Product - BEA tabela 1.1.5, linha 1. índice de preço Gross Domestic Product - BEA tabela 1.1.4, linha 1. população dos Estados Unidos - BEA tabela 2.1, linha 40.

${ }^{3}$ Foram considerados também o Airline Fares disponibilizado pelo BLS do período de 1989M1-2017M2, o Round-Trip disponibilizado anualmente pelo Airlines for America do período de 1979-2016 e o Travel Price Index (TPI) disponibilizado mensalmente pelo U.S. Travel Association no período de 1985M1-2016M12 (detalhes no site: https://www.ustravel.org/research/travel-price-index), entretanto essas séries tinham como desvantagem abranger um período menor ou ser de frequência anual.

${ }^{4}$ fonte: air transportation - BEA tabela 2.4.4 linha 74 .
} 
sua peculiaridade. Um primeiro exemplo disso é que alguns estados legalizaram apenas cassinos que fossem gerenciados por tribos indígenas, outros permitiram que os cassinos fossem gerenciados por tribos indígenas e por grupos comercias. Um segundo exemplo é que alguns estados legalizaram os cassinos sem impor restrições, outros legalizaram os cassinos impondo restrições e ao longo do tempo flexibilizaram algumas dessas restrições. Devido a essas diferenças, $L C_{t}$ considera "legalização" a data na qual o cassino foi legalizado ou a data na qual houve alguma flexibilização relevante. A tabela C.1, que está nos anexos, apresenta essas datas. 
C

\section{Datas de legalização dos cassinos}

Tabela C.1: Legalização dos cassinos

\begin{tabular}{|c|c|c|}
\hline Estado & Data & Evento "legalização" \\
\hline Nevada $^{1}$ & março de 1931 & $\begin{array}{l}\text { O governador assinou um termo de legaliza- } \\
\text { ção dos cassinos comerciais. }\end{array}$ \\
\hline New Jersey ${ }^{2}$ & novembro de 1976 & Aprovação por emenda. \\
\hline Minnesota ${ }^{3}$ & outubro de 1989 & O primeiro cassino indígena foi autorizado a operar. \\
\hline Illinois $^{4}$ & janeiro de 1990 & O estado legalizou apostas em cassinos comerciais \\
\hline Mississipi $^{5}$ & março de 1990 & $\begin{array}{l}\text { O governador do estado assinou uma lei que permitia } \\
\text { apostas em cassinos comerciais }\end{array}$ \\
\hline Colorado $^{6}$ & novembro de 2008 & $\begin{array}{l}\text { O limite máximo por aposta passou de } 5 \text { para } 100 \\
\text { dólares }\end{array}$ \\
\hline Louisiana $^{7}$ & julho de 1991 & $\begin{array}{l}\text { O Legislativo da Louisiana votou para permitir jogos } \\
\text { de cassino em embarcações }\end{array}$ \\
\hline Washington ${ }^{8}$ & setembro de 1991 & $\begin{array}{l}\text { Data na qual o estado, no qual não há cassinos } \\
\text { comerciais, assinou o primeiro acordo que autorizava } \\
\text { a operação de casino indígena }\end{array}$ \\
\hline Connecticut $^{9}$ & maio de 1991 & O primeiro cassino indígena foi autorizado a operar. \\
\hline Indiana $^{10}$ & julho de 1993 & O estado de legalizou apostas em cassinos comerciais \\
\hline Michigan $^{11}$ & novembro de 1996 & $\begin{array}{l}\text { Através de uma consulta a população, o estado lega- } \\
\text { lizou apostas em casinos comerciais. }\end{array}$ \\
\hline New Mexico & fevereiro de 1995 & O primeiro cassino indígena foi autorizado a operar. \\
\hline Missouri $^{12}$ & novembro de 1998 & $\begin{array}{l}\text { Em novembro de } 1992 \text { foram legalizadas apostas em } \\
\text { cassinos comercias do tipo navio no estado com a } \\
\text { restrição de que os navios fossem obrigados a navegar } \\
\text { durante as apostas. Essa restrição foi flexibilizada } \\
\text { através de consulta à população em novembro de } \\
\text { 1998, data considerada por } L C_{t} \text { como "legalização" }\end{array}$ \\
\hline South Dakota ${ }^{13}$ & novembro de 2000 & $\begin{array}{l}\text { Em novembro de } 1998 \text { o estado legalizou apostas } \\
\text { limitando a } 5 \text { dólares o valor máximo apostado, mas } \\
\text { em novembro de } 2000 \text { flexibilizou esse limite para } 100 \\
\text { dólares data considerada por } L C_{t} \text { como "legalização". }\end{array}$ \\
\hline
\end{tabular}

Nota: A tabela informa a data de legalização de cada cassino e o evento de "legalização'" 
D

\section{Capacidade preditiva de ptr e ptrc utilizando os modelos apresentados na tabela 2.3}

D.1

DOLS - 0

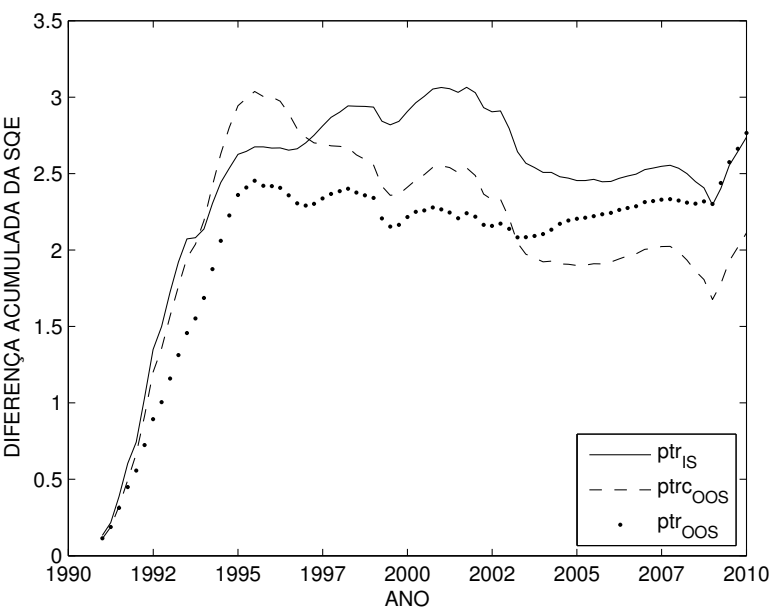

Figura D.1: Performance de $\widehat{p t r}_{t}$ e $\widehat{p t r} c_{t}$ na previsão do retorno de 24 trimestres do prêmio de risco do índice CRSP value-weighted

Tabela D.1: Previsão do prêmio de risco do índice CRSP value-weighted

\begin{tabular}{|c|c|c|c|c|c|c|c|}
\hline \multicolumn{8}{|c|}{ Horizonte de previsão em trimestres } \\
\hline previsor & estatística & 4 & 8 & 12 & 16 & 20 & 24 \\
\hline \multirow{6}{*}{$\operatorname{ptr}_{t}$} & $R^{2}$ & 2,39 & 4,41 & 4,88 & 4,85 & 10,46 & 15,45 \\
\hline & $R_{I S}^{2}$ & 5,29 & 8,06 & 9,71 & 11,47 & 19,28 & 23,63 \\
\hline & $R_{O O S}^{2}$ & $-1,04$ & 3,65 & 11,19 & 15,84 & 25,23 & 32,01 \\
\hline & $R_{O O S, C O E F}^{2}$ & 1,35 & 2,72 & 3,82 & 5,61 & 17,87 & 27,10 \\
\hline & $R_{O O S, P R E M I O}^{2}$ & $-1,04$ & 3,65 & 11,19 & 15,84 & 25,23 & 32,01 \\
\hline & $R_{O O S, C O E F, P R E M I O}^{2}$ & 1,35 & 2,72 & 3,82 & 5,61 & 17,87 & 27,10 \\
\hline \multirow{4}{*}{$\widehat{p t r c} c_{t}$} & $R_{O O S}^{2}$ & 1,60 & 5,71 & 9,46 & 10,24 & 18,42 & 16,66 \\
\hline & $R_{O O S, C O E F}^{2}$ & 2,68 & 7,17 & 11,14 & 13,33 & 19,21 & 16,83 \\
\hline & $R_{O O S, P R E M I O}^{2}$ & 1,60 & 5,71 & 9,46 & 10,24 & 18,42 & 16,66 \\
\hline & $R_{O O S, C O E F, P R E M I O}^{2}$ & 2,68 & 7,17 & 11,14 & 13,33 & 19,21 & 16,83 \\
\hline
\end{tabular}


Apêndice D. Capacidade preditiva de ptr e ptrc utilizando os modelos apresentados na tabela 2.3

D.2

DOLS - 1

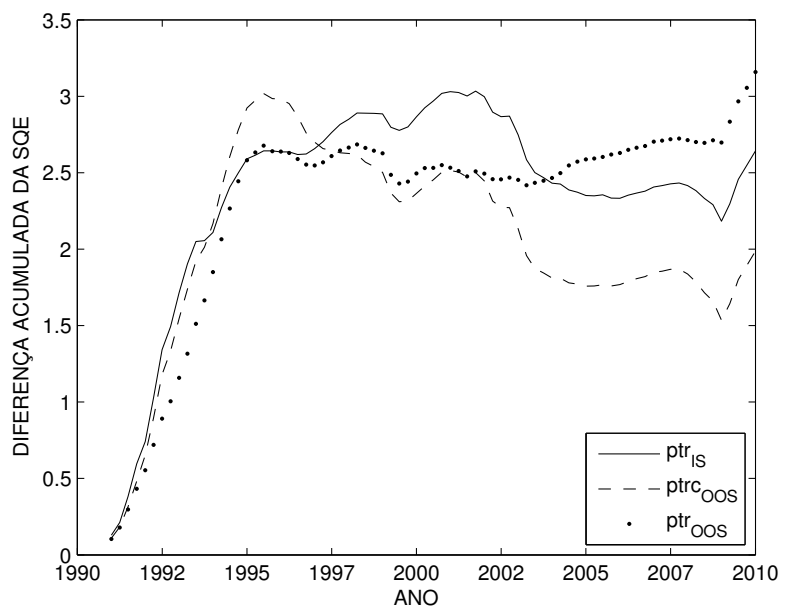

Figura D.2: Performance de $\widehat{p t r}_{t}$ e $\widehat{p t r} c_{t}$ na previsão do retorno de 24 trimestres do prêmio de risco do índice CRSP value-weighted

Tabela D.2: Previsão do prêmio de risco do índice CRSP value-weighted

Horizonte de previsão em trimestres

\begin{tabular}{cccccccc}
\hline previsor & estatística & 4 & 8 & 12 & 16 & 20 & 24 \\
\hline \multirow{6}{*}{$\widehat{p t r}_{t}$} & $R^{2}$ & 2,39 & 4,41 & 4,88 & 4,85 & 10,46 & 15,45 \\
& $R_{I S}^{2}$ & 5,29 & 8,06 & 9,71 & 11,47 & 19,28 & 23,63 \\
& $R_{\text {OOS }}^{2}$ & $-1,04$ & 3,65 & 11,19 & 15,84 & 25,23 & 32,01 \\
& $R_{\text {OOS,COEF }}^{2}$ & 1,35 & 2,72 & 3,82 & 5,61 & 17,87 & 27,10 \\
& $R_{\text {OOS,PREMIO }}^{2}$ & $-1,04$ & 3,65 & 11,19 & 15,84 & 25,23 & 32,01 \\
& $R_{\text {OOS,COEF,PREMIO }}^{2}$ & 1,35 & 2,72 & 3,82 & 5,61 & 17,87 & 27,10 \\
\hline \multirow{2trc}{*}{$\widehat{p a O S}_{t}$} & $R_{\text {OOS }}^{2}$ & 1,60 & 5,71 & 9,46 & 10,24 & 18,42 & 16,66 \\
& $R_{\text {OOS,COEF }}^{2}$ & 2,68 & 7,17 & 11,14 & 13,33 & 19,21 & 16,83 \\
& $R_{\text {OOS,PREMIO }}^{2}$ & 1,60 & 5,71 & 9,46 & 10,24 & 18,42 & 16,66 \\
& $R_{\text {OOS,COEF,PREMIO }}^{2}$ & 2,68 & 7,17 & 11,14 & 13,33 & 19,21 & 16,83 \\
\hline
\end{tabular}


Apêndice D. Capacidade preditiva de ptr e ptrc utilizando os modelos apresentados na tabela 2.3

D.3

DOLS - 2

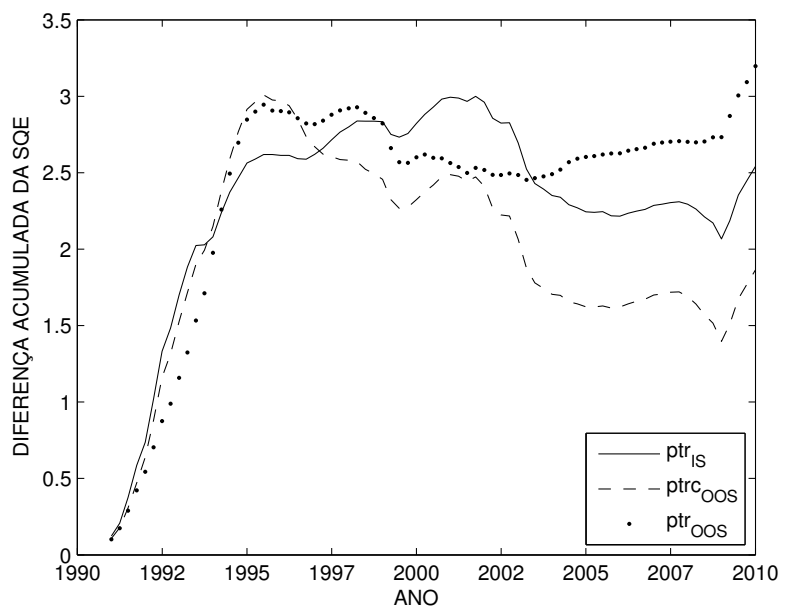

Figura D.3: Performance de $\widehat{p t r}_{t}$ e $\widehat{p t r} c_{t}$ na previsão do retorno de 24 trimestres do prêmio de risco do índice CRSP value-weighted

Tabela D.3: Previsão do prêmio de risco do índice CRSP value-weighted

Horizonte de previsão em trimestres

\begin{tabular}{cccccccc}
\hline previsor & estatística & 4 & 8 & 12 & 16 & 20 & 24 \\
\hline \multirow{6}{*}{$\widehat{p t r}_{t}$} & $R^{2}$ & 2,39 & 4,41 & 4,88 & 4,85 & 10,46 & 15,45 \\
& $R_{I S}^{2}$ & 5,29 & 8,06 & 9,71 & 11,47 & 19,28 & 23,63 \\
& $R_{\text {OOS }}^{2}$ & $-1,04$ & 3,65 & 11,19 & 15,84 & 25,23 & 32,01 \\
& $R_{\text {OOS,COEF }}^{2}$ & 1,35 & 2,72 & 3,82 & 5,61 & 17,87 & 27,10 \\
& $R_{\text {OOS,PREMIO }}^{2}$ & $-1,04$ & 3,65 & 11,19 & 15,84 & 25,23 & 32,01 \\
& $R_{\text {OOS,COEF,PREMIO }}^{2}$ & 1,35 & 2,72 & 3,82 & 5,61 & 17,87 & 27,10 \\
\hline \multirow{2trc}{*}{$\widehat{p a O S}_{t}$} & $R_{\text {OOS }}^{2}$ & 1,60 & 5,71 & 9,46 & 10,24 & 18,42 & 16,66 \\
& $R_{\text {OOS,COEF }}^{2}$ & 2,68 & 7,17 & 11,14 & 13,33 & 19,21 & 16,83 \\
& $R_{\text {OOS,PREMIO }}^{2}$ & 1,60 & 5,71 & 9,46 & 10,24 & 18,42 & 16,66 \\
& $R_{\text {OOS,COEF,PREMIO }}^{2}$ & 2,68 & 7,17 & 11,14 & 13,33 & 19,21 & 16,83 \\
\hline
\end{tabular}


Apêndice D. Capacidade preditiva de ptr e ptrc utilizando os modelos apresentados na tabela 2.3

D.4

DOLS - 3

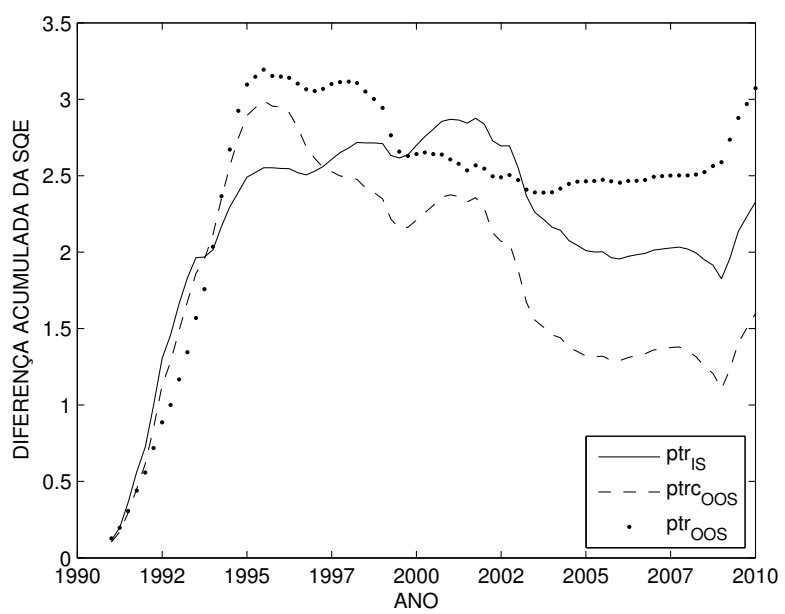

Figura D.4: Performance de $\widehat{p t r}_{t}$ e $\widehat{p t r} c_{t}$ na previsão do retorno de 24 trimestres do prêmio de risco do índice CRSP value-weighted

Tabela D.4: Previsão do prêmio de risco do índice CRSP value-weighted

Horizonte de previsão em trimestres

\begin{tabular}{cccccccc}
\hline previsor & estatística & 4 & 8 & 12 & 16 & 20 & 24 \\
\hline \multirow{6}{*}{$\widehat{p t r}_{t}$} & $R^{2}$ & 2,39 & 4,41 & 4,88 & 4,85 & 10,46 & 15,45 \\
& $R_{I S}^{2}$ & 5,29 & 8,06 & 9,71 & 11,47 & 19,28 & 23,63 \\
& $R_{\text {OOS }}^{2}$ & $-1,04$ & 3,65 & 11,19 & 15,84 & 25,23 & 32,01 \\
& $R_{\text {OOS,COEF }}^{2}$ & 1,35 & 2,72 & 3,82 & 5,61 & 17,87 & 27,10 \\
& $R_{\text {OOS,PREMIO }}^{2}$ & $-1,04$ & 3,65 & 11,19 & 15,84 & 25,23 & 32,01 \\
& $R_{\text {OOS,COEF,PREMIO }}^{2}$ & 1,35 & 2,72 & 3,82 & 5,61 & 17,87 & 27,10 \\
\hline \multirow{2trc}{*}{$\widehat{p a O S}_{t}$} & $R_{\text {OOS }}^{2}$ & 1,60 & 5,71 & 9,46 & 10,24 & 18,42 & 16,66 \\
& $R_{\text {OOS,COEF }}^{2}$ & 2,68 & 7,17 & 11,14 & 13,33 & 19,21 & 16,83 \\
& $R_{\text {OOS,PREMIO }}^{2}$ & 1,60 & 5,71 & 9,46 & 10,24 & 18,42 & 16,66 \\
& $R_{\text {OOS,COEF,PREMIO }}^{2}$ & 2,68 & 7,17 & 11,14 & 13,33 & 19,21 & 16,83 \\
\hline
\end{tabular}

